An investigation of the liquid to glass transition using integral equations for the pair structure of coupled replicae

Jean-Marc Bomont', Jean-Pierre Hansen' , and Giorgio Pastore'

Citation: The Journal of Chemical Physics 141, 174505 (2014); doi: 10.1063/1.4900774

View online: http://dx.doi.org/10.1063/1.4900774

View Table of Contents: http://aip.scitation.org/toc/jcp/141/17

Published by the American Institute of Physics

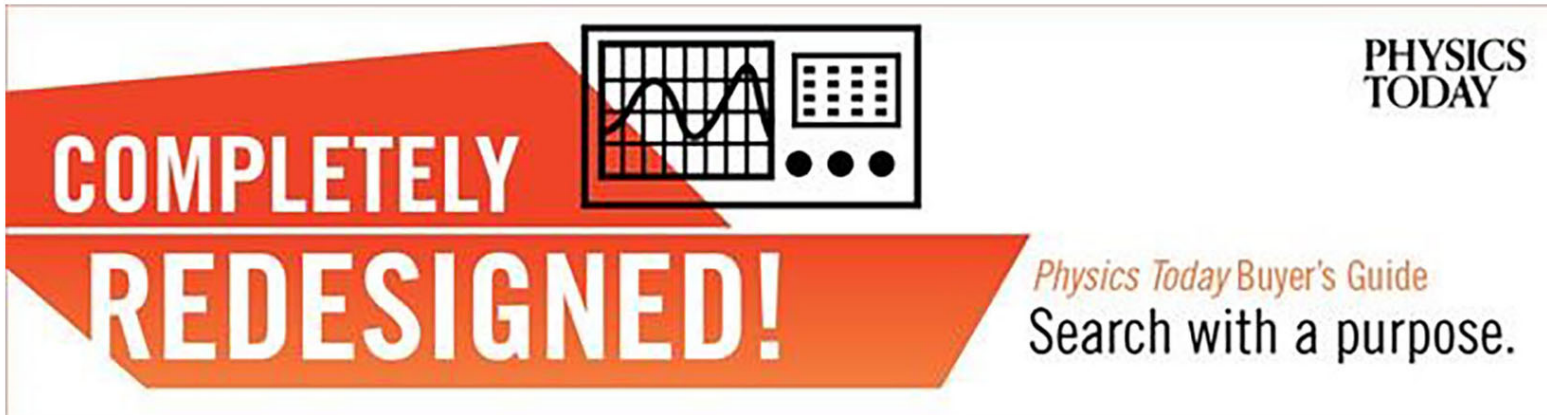




\title{
An investigation of the liquid to glass transition using integral equations for the pair structure of coupled replicae
}

\author{
Jean-Marc Bomont, ${ }^{1, a)}$ Jean-Pierre Hansen, ${ }^{2, b)}$ and Giorgio Pastore ${ }^{3, c)}$ \\ ${ }^{1}$ Université de Lorraine, LCP-A2MC, EA 3469, 1 Bd. François Arago, Metz F-57078, France \\ ${ }^{2}$ Université Pierre et Marie Curie, UMR 8234 PHENIX, Paris, France and Department of Chemistry, \\ University of Cambridge, Cambridge CB2 1EW, United Kingdom \\ ${ }^{3}$ Dipartimento di Fisica, Università di Trieste, Strada Costiera 11, 34151 Grignano, Trieste, Italy
}

(Received 16 August 2014; accepted 20 October 2014; published online 6 November 2014)

\begin{abstract}
Extensive numerical solutions of the hypernetted-chain (HNC) and Rogers-Young (RY) integral equations are presented for the pair structure of a system of two coupled replicae (1 and 2) of a "soft-sphere" fluid of atoms interacting via an inverse-12 pair potential. In the limit of vanishing inter-replica coupling $\varepsilon_{12}$, both integral equations predict the existence of three branches of solutions: (1) A high temperature liquid branch $(L)$, which extends to a supercooled regime upon cooling when the two replicae are kept at $\varepsilon_{12}=0$ throughout; upon separating the configurational and vibrational contributions to the free energy and entropy of the $L$ branch, the Kauzmann temperature is located where the configurational entropy vanishes. (2) Starting with an initial finite coupling $\varepsilon_{12}$, two "glass" branches $G_{1}$ and $G_{2}$ are found below some critical temperature, which are characterized by a strong remnant spatial inter-replica correlation upon taking the limit $\varepsilon_{12} \rightarrow 0$. Branch $G_{2}$ is characterized by an increasing overlap order parameter upon cooling, and may hence be identified with the hypothetical "ideal glass" phase. Branch $G_{1}$ exhibits the opposite trend of increasing order parameter upon heating; its free energy lies consistently below that of the $L$ branch and above that of the $G_{2}$ branch. The free energies of the $L$ and $G_{2}$ branches are found to intersect at an alleged "random first-order transition" (RFOT) characterized by weak discontinuities of the volume and entropy. The Kauzmann and RFOT temperatures predicted by RY differ significantly from their HNC counterparts. (C) 2014 AIP Publishing LLC. [http://dx.doi.org/10.1063/1.4900774]
\end{abstract}

\section{INTRODUCTION}

When a liquid is quenched sufficiently rapidly below its freezing temperature $T_{\mathrm{f}}$, so as to avoid crystallization, the supercooled liquid will go through a number of metastable states before eventually forming a long-lived glass (or an amorphous solid) phase. The phenomenology of the observed transformation is well established thanks to numerous calorimetric, dielectric relaxation, shear relaxation, nuclear magnetic resonance (NMR), or neutron scattering experiments on a wide range of materials, ${ }^{1,2}$ but a complete theoretical understanding is still lacking despite a surge in recent progress. ${ }^{3}$ The generally accepted scenario observed upon lowering the temperature is the following. At a temperature $T_{\mathrm{c}}<T_{\mathrm{f}}$, a kinetic glass transition towards a dynamically arrested state is predicted by mode coupling theory, ${ }^{4}$ but ergodicity is eventually restored by activated dynamical processes. Calorimetric measurements on a broad variety of supercooled liquids point to a rapid change in the slope of the molar volume or enthalpy as a function of temperature, at a calorimetric glass transition temperature $T_{\mathrm{g}}<T_{\mathrm{c}}$, leading to a peak in the specific heat $C_{\mathrm{p}} .{ }^{1,2}$ However, $T_{\mathrm{g}}$ is not an intrinsic property of the material, since it depends on the cooling rate; $T_{\mathrm{g}}$ decreases when the liquid

\footnotetext{
${ }^{a)}$ Electronic mail: jean-marc.bomont@univ-lorraine.fr

b)Electronic mail: jph32@cam.ac.uk

c) Electronic mail: pastore@ts.infn.it
}

is cooled more slowly. The "calorimetric glass transition" is hence not a genuine phase transition: $T_{\mathrm{g}}$ merely signals the temperature at which the structural relaxation time becomes comparable to experimental time scales (typically of the order of minutes or hours). A related, implicit definition of $T_{\mathrm{g}}$ is provided by equating the Maxwell relaxation time $\tau_{\mathrm{M}}=\eta / G_{\infty}$ (where $\eta$ is the shear viscosity and $G_{\infty}$ is the instantaneous shear modulus) and a conventional experimental time scale $\tau_{\text {exp }}=10^{3} \mathrm{~s}$. With a shear modulus $G_{\infty}=10^{9} \mathrm{Nm}^{-2}$, typical of most materials, this leads to an operational definition of $T_{\mathrm{g}}$ as being the temperature at which the shear viscosity reaches the value $\eta=10^{12} \mathrm{Nm}^{-2} \mathrm{~s}$ (or $10^{13}$ poise) $)^{5,6}$

At temperatures $T<T_{\mathrm{g}}$, the supercooled liquid does not equilibrate on reasonable experimental time scales, while extrapolation of calorimetric data leads to the expectation of a Kauzmann "entropy crisis" at a temperature $T=T_{\mathrm{K}}$, where the configurational entropy (or "complexity"), $S_{\text {conf }}$ (which will be defined later), of the supercooled liquid appears to vanish. ${ }^{7}$ A possible connection between the vanishing of $S_{\text {conf }}$ and the divergence of the structural relaxation time in strongly supercooled liquids, as embodied, e.g., in the phenomenological Vogel-Fulcher-Tamman relation for highly viscous liquids (namely, $\eta=\eta_{0} \exp \left\{-A /\left(T-T_{0}\right)\right\}$ ) was put forward in a pioneering paper by Adam and Gibbs, ${ }^{8}$ which suggests that $T_{0}=T_{\mathrm{K}}$.

An appealing conjecture postulates that the Kauzmann "entropy crisis" is pre-empted by an equilibrium phase 
transition between the supercooled liquid and an "ideal glass" phase corresponding to the lowest, non-crystalline minimum in the temperature dependent free-energy landscape. ${ }^{2,9}$ The ideal glass is characterized by a unique disordered configuration of non-periodic equilibrium positions $\left\{\mathbf{R}_{i}\right\}(1 \leq i \leq N)$ around which the $N$ atoms vibrate. Such a "random first order phase transition" (RFOT) of structural glasses has many formal analogies with mean field theories of spin glass models without intrinsic randomness. ${ }^{3}$ Concepts and methodologies developed for the latter have been successfully adapted to the former, in particular the replica method. ${ }^{10-12}$ Coupled replicæ (or "clones") of a given many-body system are introduced to provide an order parameter (the configurational "overlap") which allows a clear-cut distinction between supercooled liquid and ideal glass states. Such states are not distinguishable by an obvious broken symmetry; in particular, their disordered microscopic structures look very similar, as opposed to the periodic structure of a crystalline solid.

In the simplest and physically most transparent case, one considers $m=2$ weakly coupled replicæ (the original system and one clone) and one examines the static inter-replica correlations. ${ }^{11}$ The RFOT is then characterized by a discontinuous jump in the overlap order parameter arising from the fact that in the ideal glass state, configurations of the tworeplicæ are localized close to a unique, disordered equilibrium configuration $\left\{\mathbf{R}_{i}\right\}$. From a free energy landscape point of view, replicæ are "confined" to the basin associated with the lowest (non-crystalline) minimum. The equilibrium thermodynamics of the ideal glass can be calculated by minimizing the approximate hypernetted chain (HNC) free energy functional of Morita and Hiroike, ${ }^{13}$ which is amenable to an analytic continuation to real (rather than merely integer) values of $m$, and taking the limit $m \rightarrow 1 .^{10,12}$ This elegant approach has been first applied to soft repulsive core systems ${ }^{12}$ and their mixtures, ${ }^{14}$ and extended to the case of the hard sphere model, where the glass transition is akin to a jamming transition. ${ }^{15}$ The main drawback of the MézardParisi approach is that HNC theory of the pair structure and thermodynamics of classical fluids is known to be thermodynamically inconsistent and increasingly inaccurate at high densities. $^{6}$

In an attempt to overcome this deficiency, we return to the two-replicæ $(m=2)$ case, and numerically solve the HNC integral equation as well as its thermodynamically self-consistent extension, the Rogers-Young (RY) integral equation ${ }^{16}$ for the pair structure of the symmetric "binary mixture" of two weakly coupled replicæ over a wide range of thermodynamic states. We show the existence of three branches of solutions for the pair structure of the tworeplicæ system in the limit of vanishing inter-replica coupling, within both integral equations. Calculations of the resulting free energies and configurational overlaps allows us to locate a tentative first order transition between supercooled liquid and ideal glass states. It is important to stress that the present approach provides no clue of the underlying relaxation processes. Integral equations provide only purely static information corresponding to fully equilibrated, stable, or metastable states achieved in the limit of infinite time.
The present work complements and extends the results of an earlier, preliminary communication. ${ }^{17}$

\section{THE TWO-REPLICAE SYSTEM}

The present investigation focuses on the "soft-sphere" system of atoms interacting via the purely repulsive potential

$$
v(r)=\varepsilon\left(\frac{\sigma}{r}\right)^{12},
$$

where $\varepsilon$ is the energy scale and $\sigma$ is the length scale; we will systematically use reduced variables, in particular the reduced distance $x=r / \sigma$, the reduced number density $\rho^{*}=N \sigma^{3} / V$ (where $N$ and $V$ are the total number of particles and the total volume), and the reduced temperature $T^{*}=k_{\mathrm{B}} T / \varepsilon$. The model has two advantages: First all its dimensionless excess (i.e., non-ideal) thermodynamic properties are functions of the single dimensionless variable

$$
\Gamma=\frac{\rho^{*}}{T^{* 1 / 4}},
$$

and not of $\rho^{*}$ and $T^{*}$ separately. ${ }^{18}$ Thus, e.g., the excess Helmholtz free energy per particle is given by the universal function

$$
\frac{F^{e x}(\rho, T)}{N k_{B} T}=f_{e x}(\Gamma)
$$

while the pair distribution function $g(x)$ obeys the scaling relation (which leaves $\Gamma$ unchanged)

$$
g\left(x ; \rho^{*} ; T^{*}\right)=g\left(\lambda x ; \rho^{*} / \lambda^{3} ; T^{*} / \lambda^{12}\right),
$$

where $\lambda$ is an arbitrary scaling factor. ${ }^{18}$ The second advantage of the soft-sphere model (1) is that its equilibrium and transport properties are well documented by extensive computer simulations. In particular, the fluid freezes into a face centered cubic (FCC) crystal at $\Gamma_{\mathrm{f}}=1.15,{ }^{18,19}$ while properties of the supercooled fluid were investigated by Molecular Dynamics (MD) simulations, ${ }^{20,21}$ the MD data are of course strongly dependent on the applied cooling rate. Moreover, the pioneering replica-based investigations of the RFOT were based on the same soft-sphere model. ${ }^{10,12}$

We now consider two weakly coupled replicæ (labelled 1 and 2) of the soft-sphere system, each consisting of $N$ particles which interact via pair potentials $v_{11}(x) \equiv v_{22}(x)=v(x)$, while atoms of different replicæ interact via a weak attractive potential $v_{12}(x)$, chosen to be of the form

$$
v_{12}(x)=-\varepsilon_{12} w(x)=-\varepsilon_{12}\left[\frac{c^{2}}{x^{2}+c^{2}}\right]^{6},
$$

where $c$ is chosen such that the range of the attraction is significantly shorter than the mean distance between neighboring atoms, $d^{*}=d / \sigma \simeq \rho^{*-1 / 3}$ to ensure that, due to the strong repulsion between atoms of the same replica, an atom of one replica can interact with at most one atom of the other replica. For a non-zero $\varepsilon_{12}$, the attraction (5) induces pairing of atoms of opposite replicæ into diatomic "molecules" at sufficiently low temperatures. The precise shape of $w(x)$ is irrelevant, since we will eventually be interested in the limit $\varepsilon_{12} \rightarrow 0$. 
The total potential energy of the symmetric binary mixture of the two-replicæ reads

$$
\begin{aligned}
V_{N, N}\left(\left\{\mathbf{x}_{i}^{1}\right\},\left\{\mathbf{x}_{j}^{2}\right\}\right)= & \sum_{i} \sum_{j>i} v\left(\left|\mathbf{x}_{i}^{1}-\mathbf{x}_{j}^{1}\right|\right) \\
& +\sum_{i} \sum_{j>i} v\left(\left|\mathbf{x}_{i}^{2}-\mathbf{x}_{j}^{2}\right|\right) \\
& +\sum_{i} \sum_{j} v_{12}\left(\left|\mathbf{x}_{i}^{1}-\mathbf{x}_{j}^{2}\right|\right) .
\end{aligned}
$$

The equilibrium structure of the two-replicæ system is characterized by two pair distribution functions, namely, $g_{11}(x)$ $\equiv g_{22}(x)$ and $g_{12}(x) \equiv g_{21}(x)$. Note that the attractive cross term in the potential energy (6) breaks the scale invariance of the soft-sphere system embodied in Eq. (2), i.e., the equilibrium properties of the two-replicæ system now depend on two thermodynamic variables rather on $\Gamma$ alone. Scale invariance is of course recovered for fully decoupled replicæ, i.e., for $\varepsilon_{12}$ $=0$, when cross-correlations vanish, so that $g_{12}(x)=1$ for all $x$. Note that $g_{12}(x)$ leads directly to the overlap order parameter $Q$ of the two-replicæ system, which is a measure of the mean overlap of the configurations $\left\{\mathbf{x}_{i}^{1}\right\}$ and $\left\{\mathbf{x}_{j}^{2}\right\}$ of the two replicæ. One defines the following overlap function: ${ }^{11}$

$$
q_{1,2}=\frac{1}{N} \sum_{i=1}^{N} \omega\left(\left|\mathbf{x}_{i}^{1}-\mathbf{x}_{i}^{2}\right|\right),
$$

where $\omega(x)$ is an arbitrary function chosen such that $\omega(0)=1$ (for perfect overlap) and $\omega(x)$ vanishes rapidly for $x \gtrsim 1$. The function $w(x)$ in Eq. (5) satisfies precisely this behavior, so that we will choose $\omega(x)=w(x)$. The mean overlap is

$$
Q=\left\langle q_{1,2}\right\rangle=4 \pi \rho^{*} \int_{0}^{\infty} g_{12}(x) w(x) x^{2} d x .
$$

If the two replicæ are completely uncorrelated, $g_{12}(x)=1$, and $Q$ reduces to its "random overlap" value $Q_{r}=\left(7 \pi^{2} / 128\right) \rho^{*} c^{3}$ $=\left(7 \pi^{2} / 128\right)\left(c / d^{*}\right)^{3} \ll 1$, since $c$ is chosen to be significantly less than $d^{*}$. In the supercooled liquid state $Q$ will remain close to $Q_{r}$, but the order parameter is expected to take significantly larger values in the ideal glass state, since the positions $\left\{\mathbf{x}_{i}^{1}\right\}$ and $\left\{\mathbf{x}_{j}^{2}\right\}$ of the atoms of the two replicæ remain localized close to the disordered equilibrium position $\left\{\mathbf{X}_{i}\right\}$. The RFOT is hence expected to be characterized by a discontinuous jump of the order parameter $Q$ in the thermodynamic limit. ${ }^{11}$ A related structural signature of the RFOT is the value of the inter-replica correlation function at zero separation, $g_{12}(x=0)$.

\section{INTEGRAL EQUATIONS AND SEARCH PROTOCOLS}

The required pair distribution functions $g_{11}(x)$ and $g_{12}(x)$ of the symmetric binary mixture of the two replicæ can be calculated approximately by solving the coupled integral equations resulting from the familiar OrnsteinZernike (OZ) relation ${ }^{6}$ between the pair correlation functions $h_{i j}(x)=g_{i j}(x)-1$, and the direct correlation functions

$$
\begin{aligned}
c_{i j}(x) & (1 \leq i, j \leq 2): \\
& h_{11}(x)=c_{11}(x)+\rho^{*}\left[c_{11} \otimes h_{11}(x)+c_{12} \otimes h_{12}(x)\right], \\
& h_{12}(x)=c_{12}(x)+\rho^{*}\left[c_{11} \otimes h_{12}(x)+c_{12} \otimes h_{11}(x)\right],
\end{aligned}
$$

where $\otimes$ denotes a convolution product; note that in the present two-replicæ system, $\rho^{*}$ is the reduced density of each of the two equivalent replicæ. The OZ relations (9) must be supplemented by an approximate closure relation. The HNC closure follows from minimization of the Morita-Hiroike free energy functional ${ }^{13}$ and reads ${ }^{6}$

$$
g_{i j}(x)=\exp \left[-\beta v_{i j}(x)+\gamma_{i j}(x)\right] ; 1 \leq i, j \leq 2,
$$

where $\gamma_{i j}(x)=h_{i j}(x)-c_{i j}(x)$ is the indirect correlation function. As already mentioned, the HNC equation is thermodynamically inconsistent, i.e., different thermodynamic routes (e.g., the virial and compressibility routes to the equation of state) lead to thermodynamic properties which differ significantly (typically by $10 \%$ or more) at high densities and/or low temperatures. This inconsistency can be overcome by using thermodynamically self-consistent closures. ${ }^{6,22}$ A selfconsistent closure which is well adapted to soft-sphere systems is that of Roberts and Young (RY), ${ }^{16}$

$$
\begin{aligned}
g_{i j}(x)= & \exp \left[-\beta v_{i j}(x)\right] \\
& \times\left[1+\frac{\exp \left\{f_{i j}(x) \gamma_{i j}(x)\right\}-1}{f_{i j}(x)}\right],
\end{aligned}
$$

where $f_{i j}(x)=1-\exp \left(-\alpha_{i j} x\right)$ are "switching functions" depending on the inverse range parameters $\alpha_{11}=\alpha_{22}$ and $\alpha_{12}$; the latter are adjusted to ensure thermodynamic selfconsistency between the virial and compressibility equations of state. In the limit $\alpha_{i j} \rightarrow \infty$ (i.e., $f_{i j}(x)=1$ ), the HNC closure (10) is recovered. In the following, we choose $f_{12}(x)=1$, and vary $\alpha_{11}$ to achieve the equation of state self-consistency. The coupled integral equations resulting from the combinations of the OZ relations (9) and the closure relation (10) (HNC) or (11) (RY) were solved numerically by the efficient Gillan's method $^{23,24}$ over wide ranges of the state variables, using a spacing of the real space grid of $\Delta x=0.02$ and $2^{12}$ or $2^{13}$ intervals, ensuring that the pair correlation functions have decayed to values of the order of $10^{-12}$ or less. Convergence was assumed to be achieved when the mean square difference between the correlation functions evaluated in successive iterations was low enough to ensure stability of the results at least including the fifth significant figure.

Solutions of the two integral equations were first mapped out for $\varepsilon_{12} \equiv 0$ (i.e., for a one-component, non-replicated system) by gradually increasing $\Gamma$ starting from a state in the stable liquid range $\left(\Gamma<\Gamma_{\mathrm{f}}\right)$ up to $\Gamma \simeq 2$, corresponding to a supercooled liquid (L) branch. Comparison between the $g(x)$ obtained via HNC and RY shows that the RY solution is systematically more structured than its HNC counterpart.

The two-replicæ system $\left(\varepsilon_{12}>0\right)$ is used in an attempt to locate the ideal glass branch of the equation of state. For each $\Gamma>\Gamma_{\mathrm{f}}$, solutions of the two integral equations are sought for finite values of $\varepsilon_{12}$, and the behavior of $h_{12}(x)$ and of $Q$ is examined in the limit $\varepsilon_{12} \rightarrow 0$. If the configurations of the two replicae de-correlate in that limit (i.e., if $h_{12}(x)=0$ and 

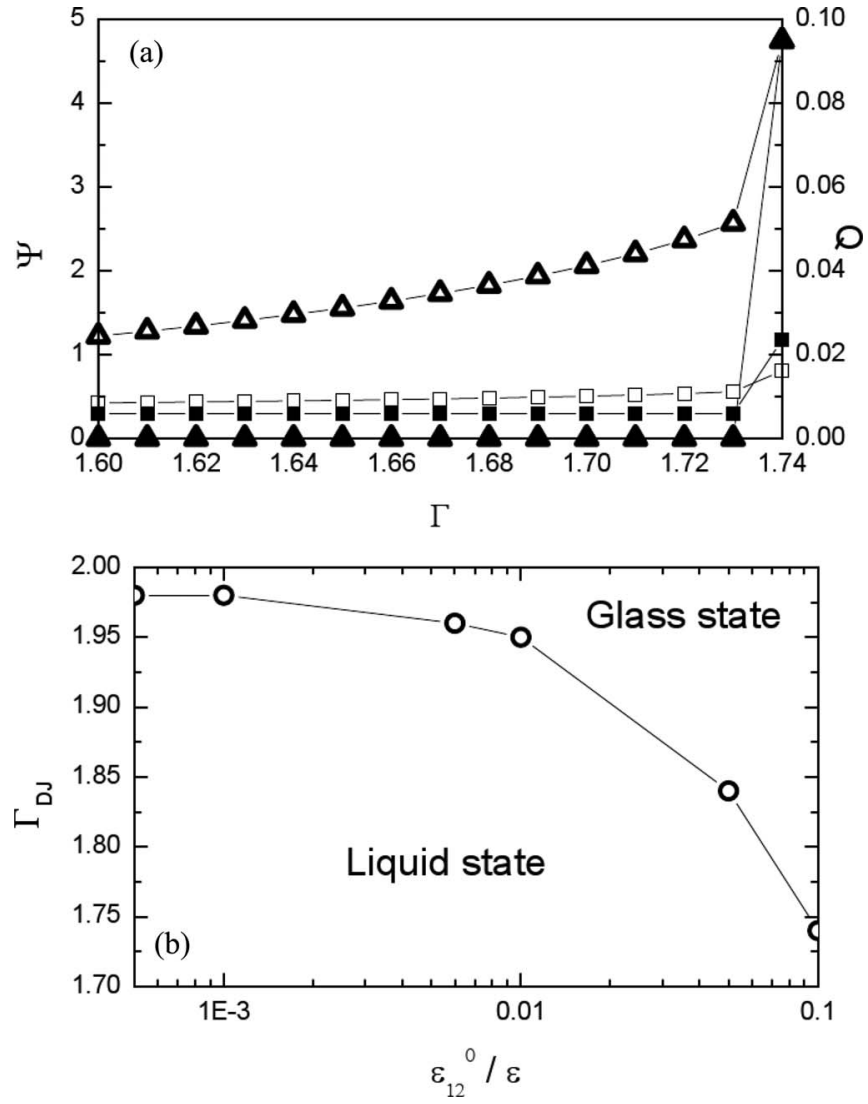

FIG. 1. (a) Order parameters $\Psi=h_{12}(x=0)$ (triangles, left-hand axis) and $Q$ (squares, right-hand axis) vs $\Gamma$ for $\varepsilon_{12}^{0}=0.1 \varepsilon$ (open triangles and squares) and once the coupling $\varepsilon_{12}$ has been switched off (full triangles and squares), as calculated from the RY integral equation; (b) RY data for $\Gamma_{\mathrm{DJ}}$ as a function of $\varepsilon_{12}^{0}$. The data point on the right end of frame (b) corresponds to the situation detailed in frame (a).

$Q=Q_{r}$ ), a supercooled liquid state is recovered. On the contrary, if there is a strong correlation between the configurations (i.e., $h_{12}(x=0) \gg 0$ and $Q \gg Q_{r}$ ), the system is regarded as being in an ideal glass state. We have adopted three different search protocols to detect the expected RFOT transition between the two states.

Protocol $\mathbf{a}$ is that used in Ref. 17. We start from an initial value $\varepsilon_{12}^{0}$ of the inter-replica coupling and gradually increase $\Gamma$. A central peak develops in $h_{12}(x)$ around $x=0$, whose amplitude grows steadily as $\Gamma$ increases, due to the increasingly strong pairing of atoms of opposite replicæ. At some critical value $\Gamma=\Gamma_{\mathrm{DJ}}$, the order parameters $\Psi(\Gamma)=h_{12}(r=0)$ and $Q(\Gamma)$ undergo a discontinuous jump to much higher values, which persists even when the coupling $\varepsilon_{12}$ is progressively switched off, while for $\Gamma<\Gamma_{\mathrm{DJ}}, \Psi \rightarrow 0$, and $Q \rightarrow Q_{r}$ as $\varepsilon_{12} \rightarrow 0$. The jumps of $\Psi$ and $\mathrm{Q}$ signal a broken translational symmetry of the two-replicæ system. An example of this behavior is illustrated in Fig. 1(a) based on RY data. Repeating the procedure for several initial values $\varepsilon_{12}^{0}$ allows us to construct a $\Gamma_{\mathrm{DJ}}$ vs $\varepsilon_{12}^{0}$ plot as illustrated in Fig. 1(b); states below the "jump" line correspond to a "molecular liquid" phase, while states above the line may be associated with a glass phase, characterized by non-zero values of $\Psi(\Gamma)$ and $Q-Q_{r}$ in the limit $\varepsilon_{12} \rightarrow 0$. After this limit has been taken, one can map out a glass branch in the $(\Gamma, Y)$ plane, where $Y$ is any structural or reduced thermodynamic property of interest, as discussed in Sec. IV. Once a glass-like solution of the HNC or RY integral equations has been obtained for a given value of $\Gamma$ via protocol a, the glass branch can also be mapped out by gradually increasing or decreasing $\Gamma$ with a small increment $\Delta \Gamma(\simeq 0.01)$; at each step, a new HNC or RY solution for $g(x)$ and $g_{12}(x)$ (with $\varepsilon_{12}=0$ ) is calculated taking the solution at the previous value of $\Gamma$ as input (initial guess). The two routes for mapping out the glass branch yield identical results for the structure and thermodynamics which will be discussed in Sec. IV.

Protocols $\mathbf{b}$ and $\mathbf{c}$ focus on a possible "smooth" transition from "molecular" liquid states to ideal glass states by starting from larger values of $\varepsilon_{12}^{0}$ (typically of order 1) leading to strongly bound pairs of atoms of opposite replicæ. In protocol $\mathbf{b}$, the two-replicæ system is first cooled for a fixed value of $\varepsilon_{12}^{0}$, but for a weaker intra-replica coupling $v(r)$, which is multiplied by a factor $\zeta<1$, so that the atoms of each replica "feel" an effective temperature $T_{\text {eff }}^{*}=T^{*} / \zeta>T^{*}$. At sufficiently low $T^{*}, \zeta$ is gradually increased (i.e., $T_{e f f}^{*}$ is decreased), and for successive values of $\zeta, \varepsilon_{12}$ is gradually reduced towards $\varepsilon_{12}=0$. A "remnant" (glass) solution characterized by $\Psi(\Gamma)>0$ and $Q>Q_{r}$ is found for $\zeta>\zeta_{c}$ (i.e., for an effective temperature $T_{\text {eff }}^{*}<T^{*} / \zeta_{c}$ ). The glass branch can thus be mapped out over a range of values of $\Gamma$ by varying $\zeta$ over the interval $\left[\zeta_{c}, 1\right]$. Applications of this protocol will be shown in Sec. IV. Protocol $\mathbf{c}$ is also designed to link the "molecular" liquid phase of the two-replicæ system to the ideal glass phase by using a $v_{12}(r)$ potential of both large amplitude $\left(\varepsilon_{12} \simeq 1\right)$ and longer range $(c \geq 0.5)$. As will be shown in Sec. IV, this protocol allows a continuous transition from "molecular" liquid to ideal glass states upon letting $\varepsilon_{12}$ go to zero.

\section{PAIR STRUCTURE AND THERMODYNAMICS}

\section{A. The one component system}

In this section, we examine the pair structure and thermodynamic properties of the supercooled liquid phase, as predicted by the HNC and RY theories of the non-replicated soft-sphere fluid $\left(\varepsilon_{12} \equiv 0\right)$ for $\Gamma>\Gamma_{\mathrm{f}}$. The excess internal energy per particle, $u_{e x}(\Gamma)=U_{e x}(\rho, T) / N k_{B} T$, equation of state $\Pi_{e x}(\Gamma)=P / \rho k_{B} T-1=4 u_{e x}(\Gamma)$ and isothermal compressibility $\chi(\Gamma)=\chi_{T} / \chi_{T}^{i d}=(\partial \beta P / \partial \rho)_{T}^{-1}$ can be calculated from the pair distribution function $g(x)$ by the standard relations. ${ }^{6}$ Within HNC theory, the excess chemical potential, $\beta \mu_{e x}$ is also expressible in terms of integrals of $h(x)$ and $c(x) ;{ }^{6}$ the excess free energy per particle (3) then follows from the standard thermodynamic relation $f_{e x}(\Gamma)=\beta \mu_{e x}(\Gamma)-\Pi_{e x}(\Gamma)$, while the excess entropy per particle is given by

$$
\begin{aligned}
s_{e x}(\Gamma) & =S_{e x} / N k_{B}=u_{e x}(\Gamma)-f_{e x}(\Gamma) \\
& =\frac{\Gamma}{4} f_{e x}^{\prime}(\Gamma)-f_{e x}(\Gamma) .
\end{aligned}
$$

The RY integral equation does not provide an expression of $\mu_{e x}$ (and hence of $f_{e x}$ ) in terms of $g(x)$ and $c(x)$ alone, contrary to $\mathrm{HNC}$ theory; $f_{e x}$ must hence be calculated by 
thermodynamic integration of the internal energy

$$
f_{e x}(\Gamma)=f_{e x}\left(\Gamma_{0}\right)+4 \int_{\Gamma_{0}}^{\Gamma} u_{e x}\left(\Gamma^{\prime}\right) \frac{d \Gamma^{\prime}}{\Gamma^{\prime}} .
$$

The initial state point $\Gamma_{0}$ may be chosen to be sufficiently low $\left(\Gamma_{0} \simeq 0.5\right.$ ) for the HNC value of $\mu_{e x}\left(\Gamma_{0}\right)$ to be quantitatively reliable.

Deep in the supercooled regime $\left(\Gamma>\Gamma_{\mathrm{f}}\right)$, atomic configurations $\left\{\mathbf{x}_{i}\right\}$ are trapped for very long times in local minima of the free energy landscape, and individual "caged" atoms vibrate around long-lived equilibrium positions $\left\{\mathbf{X}_{i}\right\}$. It is hence natural to divide the free energy and entropy into "configurational" and "vibrational" contributions ${ }^{12}$

$$
\begin{aligned}
& f=f_{i d}+f_{e x}=f_{c}+f_{v}, \\
& s=s_{i d}+s_{e x}=s_{c}+s_{v},
\end{aligned}
$$

where $s_{c}$ is referred to as "complexity" in the spin-glass literature, and determines the exponentially large number of metastable states or local minima in the free energy landscape $^{12}$

$$
\mathcal{N} \approx \exp \left\{N s_{c}\right\}
$$

An operational definition of the Kauzmann temperature $T_{\mathrm{K}}$ is that temperature at which the configurational entropy $s_{c}$ vanishes, corresponding to the situation where the system is trapped in the lowest minimum of the free energy landscape, such that $\mathcal{N}\left(T_{\mathrm{K}}\right) \simeq 1$. To determine $s_{c}$, we use $s_{e x}$, as calculated from the HNC or RY integral equations, and an estimate of $s_{v}$ based on the mean Einstein frequency $\omega_{E}$ of a disordered medium, ${ }^{6}$ as explained in Appendix A.

The resulting reduced configurational free energies $f_{c}^{*}$ $=F_{c} / N \varepsilon=f_{c}(\Gamma) T^{*}$ are plotted as functions of $T^{*}$, for fixed $\rho^{*}=1$, in Fig. 2; the corresponding configurational entropies follow from (12) or equivalently from $s_{c}=\left(\partial f_{c}^{*} / \partial T^{*}\right)_{\rho^{*}}$. The HNC and RY configurational free energies $f_{c}^{*}$ are seen to go through a maximum at a well-defined temperature where

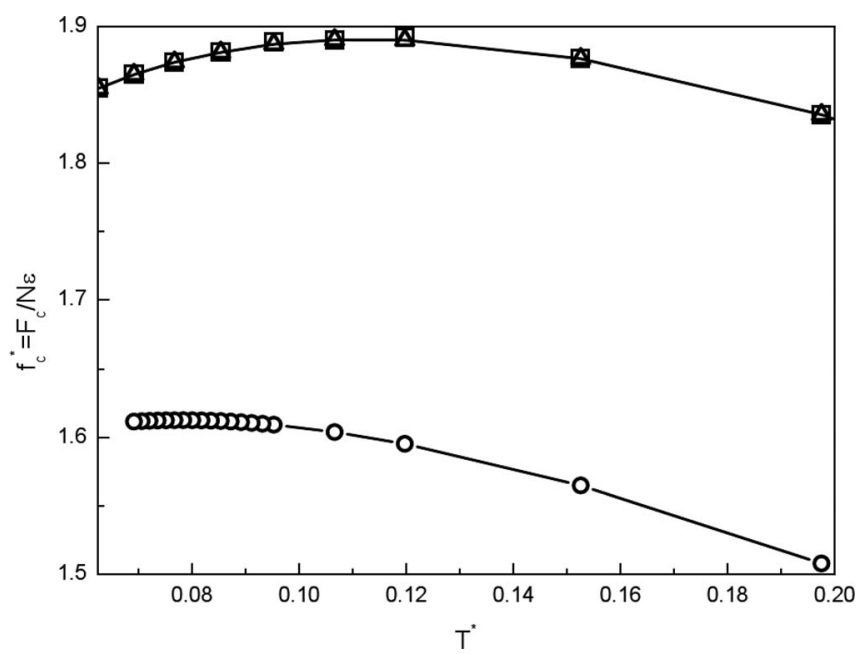

FIG. 2. HNC (triangles) and RY (circles) results for the reduced configurational free energy $f_{c}^{*}=F_{c} / N \varepsilon$ vs reduced temperature $T^{*}$, for fixed $\rho^{*}=1$. HNC results obtained from thermodynamic integration are also shown (squares). the configurational entropy vanishes. This temperature may hence be identified with the Kauzmann temperature $T_{\mathrm{K}}^{*}$, below which $s_{c}$ would take on unphysical negative values. The two estimate of the Kauzmann temperature are

$$
\begin{aligned}
T_{\mathrm{K}}^{*}(H N C) & =0.108\left(\Gamma_{\mathrm{K}}(H N C)=1.74\right), \\
T_{\mathrm{K}}^{*}(R Y) & =0.078\left(\Gamma_{\mathrm{K}}(R Y)=1.89\right) .
\end{aligned}
$$

The Kauzmann "catastrophe" is conjectured to be pre-empted by the RFOT to the "ideal glass" at some $T^{*} \gtrsim T_{\mathrm{K}}^{*}$. We hence now turn our attention to the two-replicæ system in an attempt to describe the structure and thermodynamics of the ideal glass phase.

\section{B. The two-replicae system}

To map out the thermal history of the glass phase (characterized by finite values of the order parameters $\Psi(\Gamma)$ and $Q-Q_{r}$ ), we initially adopted Protocol a presented in Sec. III. Starting from $\Gamma_{\mathrm{DJ}}\left(\varepsilon_{12}^{0} \rightarrow 0\right)=2.18$ (HNC) or 1.98 (RY) (cf. Fig. 1(b)) we solved the two integral equations for $g(x)$ and $g_{12}(x)$ (with $\varepsilon_{12}=0$ ) for gradually lower values of $\Gamma$, with a decrement $\Delta \Gamma \simeq 0.01$, and calculated $u_{e x}, Q$ and $\Psi$ for each $\Gamma$. In the case of $\mathrm{HNC}$, we also calculated $\mu_{e x}$ and $f_{e x}$ as explained above. Note that for a system of two decoupled replicæ $\left(\varepsilon_{12}=0\right)$, the excess internal energy $u_{e x}(\Gamma)$ is calculated as for a one-component system (because $v_{12}(x)=0$ ), while the HNC relation for the excess chemical potential $\mu_{e x}$ must be that adapted to a (symmetric) binary mixture (see Appendix B),

$$
\begin{aligned}
\beta \mu_{e x}(\Gamma)= & \frac{\rho^{*}}{2} \int\left[h_{11}(x) \gamma_{11}(x)-2 c_{11}(x)\right] d \mathbf{x} \\
& +\frac{\rho^{*}}{2} \int\left[h_{12}(x) \gamma_{12}(x)-2 c_{12}(x)\right] d \mathbf{x} .
\end{aligned}
$$

In the case of the RY equation, thermodynamic integration (cf. Eq. (13)) must be used; this requires the existence of a reversible thermodynamic path linking a state of the tworeplicæ system at a given value of $\Gamma$ to a state for which the excess free energy $f_{e x}$ is known. For the one-component supercooled liquid phase an obvious reference state is a high temperature/low density (i.e., low $\Gamma$ ) state, as explained earlier. But this option is not available for the ideal glass phase which becomes unstable below a critical $\Gamma$ as will become clear shortly; this problem can be overcome within the search Protocol $\mathbf{c}$ to which we will return later.

The main, unexpected result of the HNC and RY calculations of the structure and thermodynamics of the two-replicæ system following Protocol a, is the existence of two glass-like branches in the $(\Gamma, Y)$ plane, where $Y$ is the order parameter $\Psi$ or $Q$, the excess internal energy $u_{e x}$ or the excess free energy $f_{e x}$. The two branches will be referred to as $G_{1}$ and $G_{2}$. At high values of $\Gamma$, Protocol a predicts a discontinuous jump from the supercooled (L) phase to the glass phase $G_{1}$ upon taking the limit $\varepsilon_{12} \rightarrow 0$, as illustrated in Fig. 1. As $\Gamma$ is gradually lowered along the branch $G_{1}$ ("annealing") a critical state $\Gamma_{\mathrm{c}}$ is eventually reached where $\Psi, Q, u_{e x}$, and $f_{e x}$ all undergo a discontinuous jump to a new state of lower energy $u_{e x}$ and free energy $f_{e x}$ and of larger order parameters $\Psi$ and $Q$. While the 

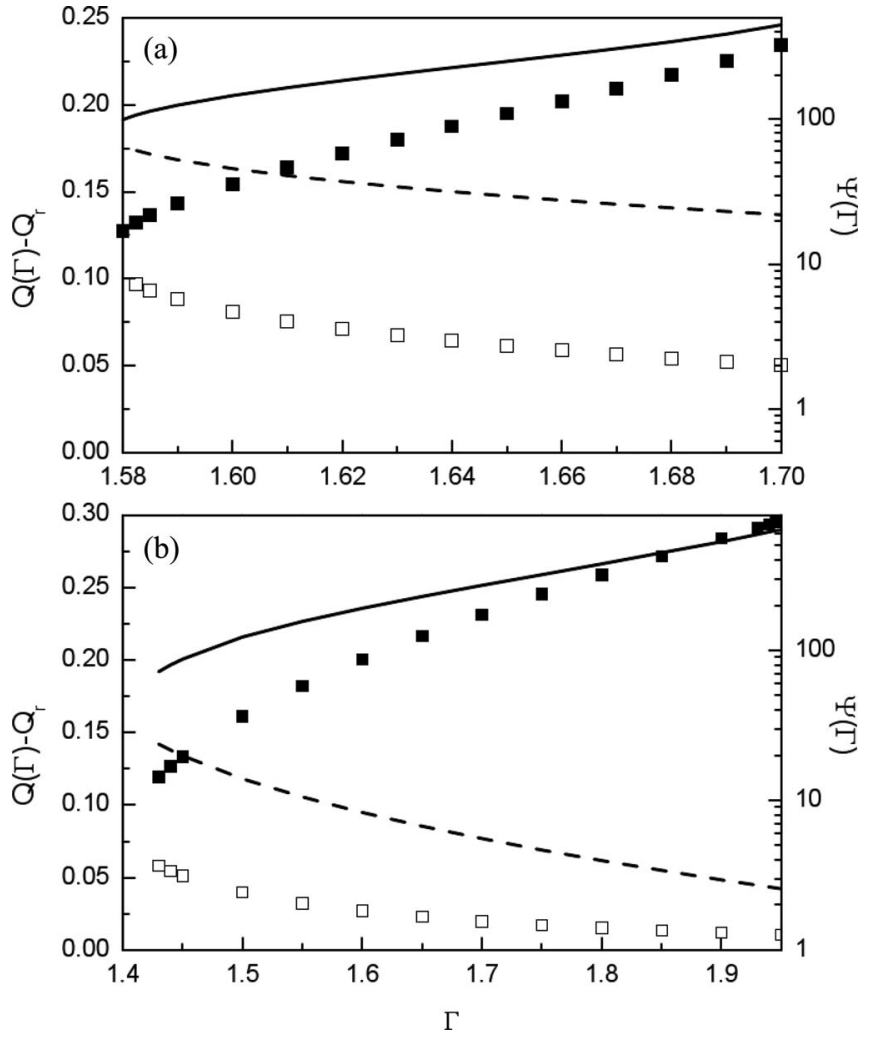

FIG. 3. Order parameters $Q(\Gamma)-Q_{r}$ (squares) and $\Psi=h_{12}(x=0)$ (line) vs $\Gamma$ along the two glass branches $G_{1}$ (open squares and dotted line) and $G_{2}$ (full squares and full line); (a) HNC results; (b) RY results.

discontinuity is of modest amplitude for the thermodynamic properties (less than 1\%) it is considerable for the order parameters (cf. Figures 3-5).

Once the lower free energy state was reached, the tworeplicæ system was cooled down, i.e., $\Gamma$ was gradually increased, and a new branch (referred to as $G_{2}$ ) was mapped out. The scenario is illustrated in Figures 3-6. Both HNC and RY predict the same qualitative scenario, but there are significant quantitative differences as shown in the figures. In particular, the critical value of the coupling, where the discontinuous jump from the branch $G_{1}$ to the branch $G_{2}$ occurs, is lower for RY $\left(\Gamma_{\mathrm{c}}=1.43\right)$ than for HNC $\left(\Gamma_{\mathrm{c}}=1.58\right)$. The key observation is that, while the order parameters $\Psi$ and $Q$ increase upon cooling (increasing $\Gamma$ ) along the branch $G_{2}$, a behavior one would intuitively expect for an ideal glass, the opposite behavior is observed along the branch $G_{1}$, where the order parameters increase upon annealing (i.e., reducing $\Gamma$ )! A possible interpretation of this counter-intuitive behavior will be suggested in the next paragraph. Fig. 6 compares the RY results for the excess reduced internal energy $u_{e x}^{*}=U_{e x} / N \varepsilon$ $=u_{e x}(\Gamma) / \Gamma^{4}$ along the three branches $\mathrm{L}, G_{1}$, and $G_{2}$ to the FCC crystal results as calculated from anharmonic phonon theory which agree within errors bars with MD data. ${ }^{20}$ The excess internal energies along the $G_{1}$ branch appear to tend towards the $u_{e x}^{*}$ values along the L branch at large $\Gamma$, while the excess internal energies along the $G_{2}$ branch are very close to their FCC counterparts for $\Gamma \simeq 1.95$. Fig. 7 compares the RY pair distribution functions $g(x)$ at $\Gamma=1.8$ for the $\mathrm{L}, G_{1}$, and $G_{2}$ phases. The amplitudes and peak positions of the three $g(x)$
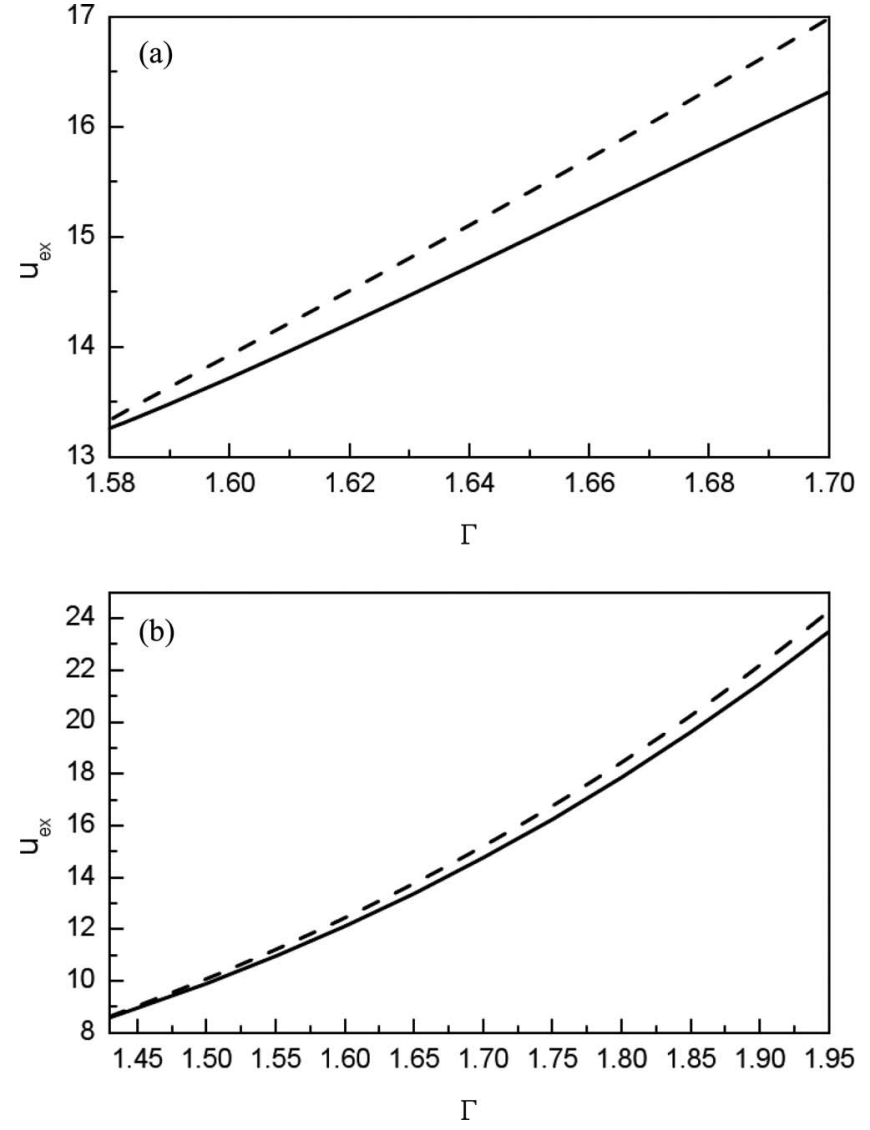

FIG. 4. Excess internal energy per particle $u_{e x}=U_{e x} /(N k T)$ vs $\Gamma$ along the glass branches $G_{1}$ (dashed line) and $G_{2}$ (full line); (a) HNC results; (b) RY results.

are very close, confirming the generally accepted view that the pair structure does not discriminate significantly between the supercooled liquid and glass phases under the same thermodynamic conditions. On the other hand, Fig. 8 illustrates the considerable differences in the inter-replica pair distribution functions $g_{12}(x)$ of the $G_{1}$ and $G_{2}$ phases under identical thermodynamic conditions, as already illustrated by the very different values of the order parameters $\Psi$ and $Q$. Note that beyond the central peak $(x>1), g_{12}(x)$ of phase $G_{2}$ is remarkably close to the pair distribution function $g(x)$, as one might expect if atoms of the two replicæ are localized near the same equilibrium positions $\left\{\mathbf{X}_{i}\right\}$ characterizing the lowest valley of the free energy landscape. The oscillations of $g_{12}(x)$ in the $G_{1}$ phase are much more strongly damped, suggesting that the atoms of the two replicæ may be trapped near slightly different equilibrium positions, corresponding to neighboring valleys of the free energy surface. The increase of the order parameters $\Psi$ and $Q$ upon annealing the phase $G_{1}$ could then be understood by assuming that the additional thermal energy allows configurations associated with neighboring free energy minima to overcome the barrier separating these minima in the landscape. This, of course, is pure conjecture, but it should be stressed that the coordination number associated with the central peak of $g_{12}(x)$ is always close to 1 , confirming the "diatomic" nature of the two-replicæ system.

As explained at the end of Sec. III, states belonging to the glass phases $G_{1}$ and $G_{2}$ may be reached via alternative 

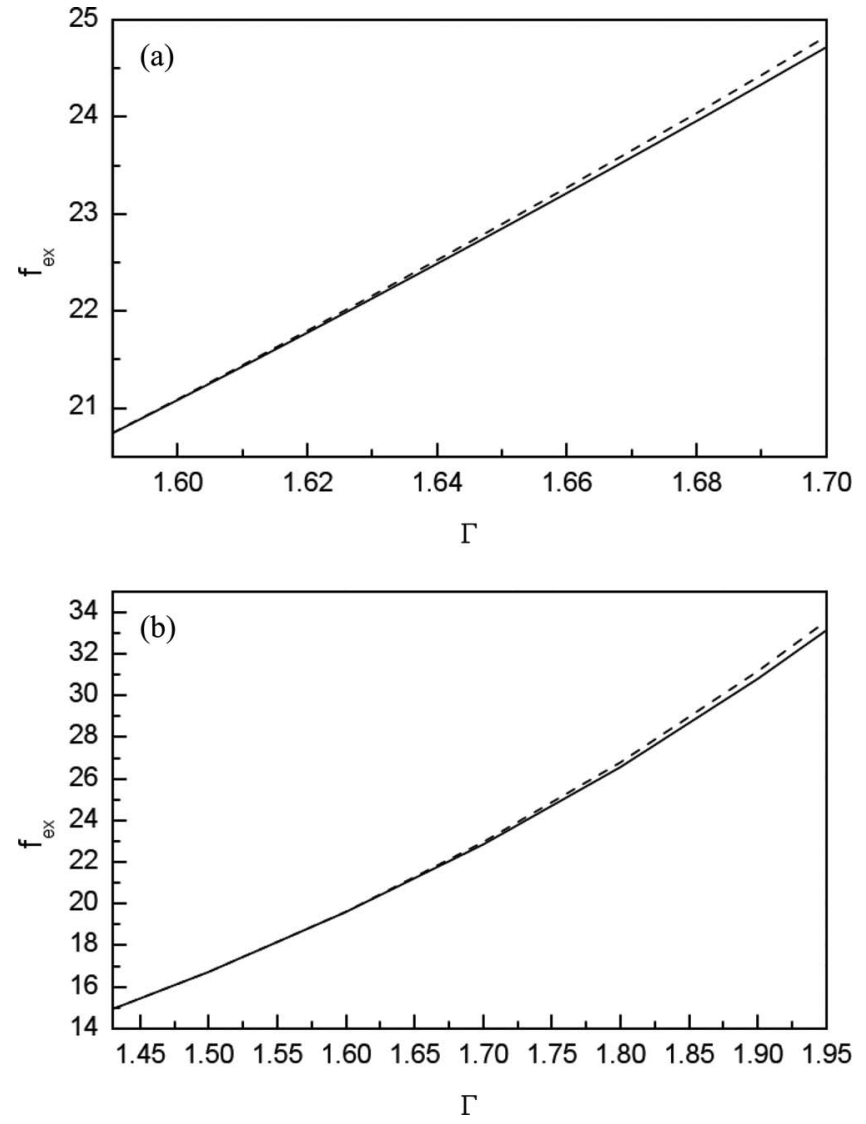

FIG. 5. Excess free energy per particle $f_{e x}$ vs $\Gamma$ along the glass branches $G_{1}$ (dashed line) and $G_{2}$ (full line); (a) HNC results; (b) RY results.

thermal histories based on Protocols $\mathbf{b}$ or $\mathbf{c}$. The thermal path from the "molecular liquid" to the glass phases is illustrated in Fig. 9 for protocol b. The "molecular liquid" with $\varepsilon_{12}^{0}=1$ and a weakened intra-replica pair potential $v_{\zeta}(r)=\zeta v(r)$ is cooled to some temperature $T^{*}$; the effective temperature "felt" by the atoms of a same replica is $T_{\text {eff }}^{*}(\zeta)=T^{*} / \zeta$. Start-

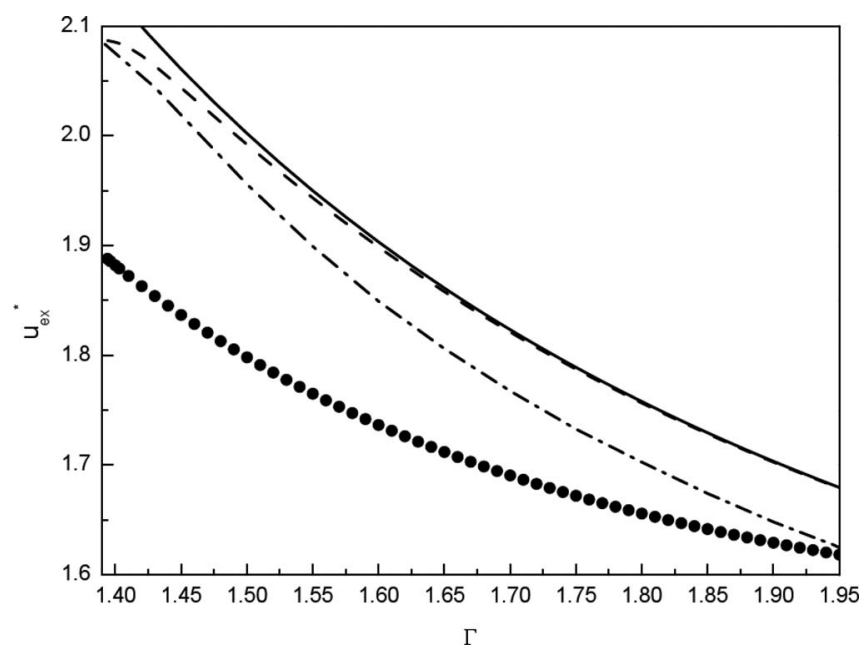

FIG. 6. RY results for the reduced excess internal energy per particle $u_{e x}^{*}$ vs $\Gamma$ : full line: supercooled liquid $(L)$ branch; dashed line: glass branch $G_{1}$; dashed-dotted line: glass branch $G_{2}$; black dots: results for the anharmonic FCC crystal.

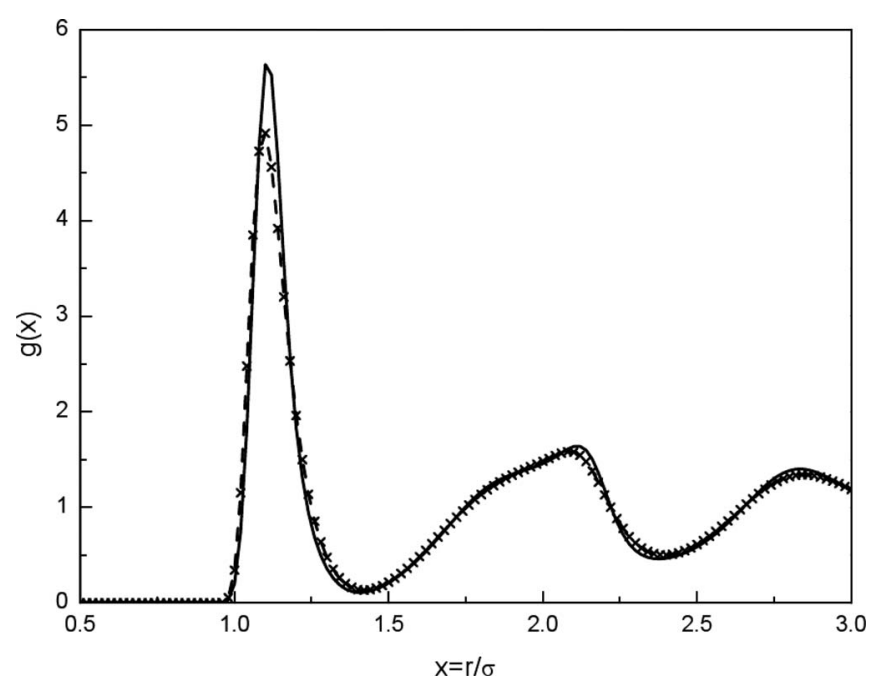

FIG. 7. $g(x)$ vs $x$ for the $\mathrm{L}$ (crosses), $G_{1}$ (dashed line), and $G_{2}$ (full line) phases at $\Gamma=1.8$, as calculated by the RY integral equation.

ing from a given $T_{e f f}^{*}(\zeta), \zeta$ is gradually increased towards $\zeta$ $=1\left(T_{\text {eff }}^{*}=T^{*}\right)$, with a small increment $\Delta \zeta=0.01$. For each value of $\zeta$, one then attempts to gradually switch off the interreplica coupling $\left(\varepsilon_{12} \rightarrow 0\right)$. For less than a threshold value $\zeta_{t}$, no solution of the HNC (or RY) integral equation for the two coupled replicæ is found for $\varepsilon_{12}$ less than a critical value $\varepsilon_{12}(\zeta)$, i.e., total extinction of the inter-replica coupling is not possible, and the system stays in the "molecular liquid" phase. At the threshold value $\zeta_{t}$, a "remnant" solution of the integral equation is found in the limit $\varepsilon_{12} \rightarrow 0$, characterized by non-zero values of the order parameters $\Psi(\Gamma)$ and $Q-Q_{r}$. Keeping now $\varepsilon_{12}=0$, successive solutions are generated for increasing values of $\zeta$, i.e., decreasing values of $T_{e f f}^{*}$, until $\zeta$ $=1$ and $T_{\text {eff }}^{*}=T^{*}$; the final solution is identical to that found via Protocol a (glass branch $G_{1}$ ) at that temperature.

Returning now to the threshold value $\zeta_{t}$, we investigate the behavior of the two-replicæ system when inter-replica coupling is gradually switched off (starting from the same

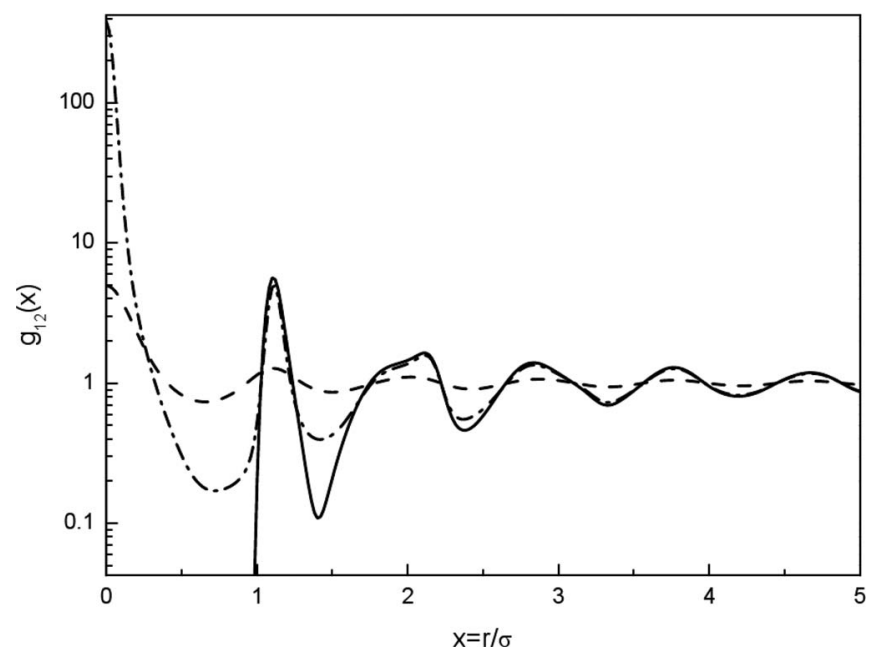

FIG. 8. RY results for the inter-replica pair distribution function $g_{12}(x)$ at $\Gamma=1.8$. Dashed line: $G_{1}$ branch; dashed-dotted line: $G_{2}$ branch; for comparison, $g(x)$ of the $G_{2}$ branch is shown as a full curve; all pair distribution functions are plotted on a logarithmic scale. 


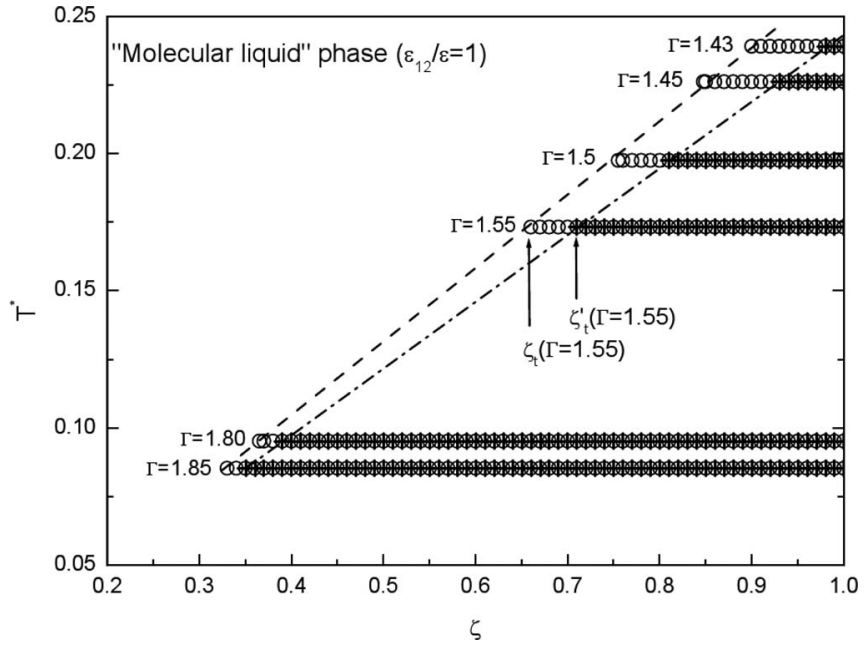

FIG. 9. Schematic representation of the thermal history followed in Protocol b with RY, linking the two glass branches $G_{1}$ and $G_{2}$ to the "molecular liquid" phase. At a sufficiently high $\Gamma$, when $\varepsilon_{12}$ is switched off for values $\zeta_{t}<\zeta<\zeta_{t}^{\prime}$, a "remnant" solution is found which eventually, in the limit where $\zeta \rightarrow 1$, leads to a state of the glass branch $G_{1}$ (circles). A state of the glass branch $G_{2}$ (crosses) is obtained in the limit $\zeta \rightarrow 1$ if $\varepsilon_{12}$ is switched off for $\zeta>\zeta_{\mathrm{t}}^{\prime}$. Values of $\zeta_{\mathrm{t}}$ and $\zeta_{\mathrm{t}}^{\prime}$ are indicated by arrows for $\Gamma=1.55$.

initial value $\varepsilon_{12}^{0}$ ) for values $\zeta>\zeta_{\mathrm{t}}$. Up to a second threshold value $\zeta_{\mathrm{t}}^{\prime}>\zeta_{\mathrm{t}}$, the same solutions are generated, leading to a state on the glass branch $G_{1}$ when $\zeta \rightarrow 1$. However, at $\zeta_{\mathrm{t}}^{\prime}$, a different "remnant" solution is found which eventually, in the limit where $\zeta \rightarrow 1$ (with $\varepsilon_{12}=0$ ), leads to a state of the glass branch $G_{2}$, identical to that obtained via protocol $\mathbf{a}$. This rather tedious procedure is now repeated for increasing values of $T^{*}$ as illustrated in Fig. 9 and the two branches $G_{1}$ and $G_{2}$ are mapped out independently of each other. Protocol b avoids the discontinuous jump between the two branches at $\Gamma=\Gamma_{\mathrm{c}}$ observed with Protocol a. The structural and thermodynamic properties at any given value of $\Gamma>\Gamma_{\mathrm{c}}$ along the two branches are identical to those generated via Protocol a, using either $\mathrm{HNC}$ or RY integral equations, thus giving us confidence that the predictions are not mere artifacts of these equations.

This observation is confirmed by calculations based on Protocol c, which is a simpler variant of Protocol b, linking the "molecular liquid" phase to the glass phases $G_{1}$ and $G_{2}$. The starting point is the "molecular liquid" phase, with a strong coupling between atoms of opposite replicæ $\left(\varepsilon_{12}^{0}=1\right)$; the key difference relative to Protocol $\mathbf{b}$ lies in the choice of a significantly larger range of $v_{12}(x)$ in Eq. (5), namely, $c=0.5$ or 1 (compared to the range parameter $c=0.225$ used in calculations within Protocols $\mathbf{a}$ and $\mathbf{b}$ ). For fixed $\varepsilon_{12}^{0}$ and $c$, the HNC or RY equation are solved for the two-replicæ system upon gradually lowering $T^{*}$ for fixed $\rho^{*}$ (conveniently chosen to be $\rho^{*}=1$ ), i.e., for increasing $\Gamma$. Once the critical values of $\Gamma$ are reached, above which the glass phases $G_{1}$ and $G_{2}$ were found to exist within Protocol $\mathbf{a}\left(\Gamma_{\mathrm{c}}=1.392\right.$ within RY) as illustrated in Figures 3-5, the inter-replica coupling $\varepsilon_{12}$ is gradually lowered towards extinction $\left(\varepsilon_{12}=0\right)$. This two-step thermodynamic path is illustrated in Fig. 10 by the variation of the order parameters $\Psi$ and $Q$, first with $\Gamma$ (frame a) and subsequently with $\varepsilon_{12}$ (frame $\mathrm{b}$ ). The final solu-
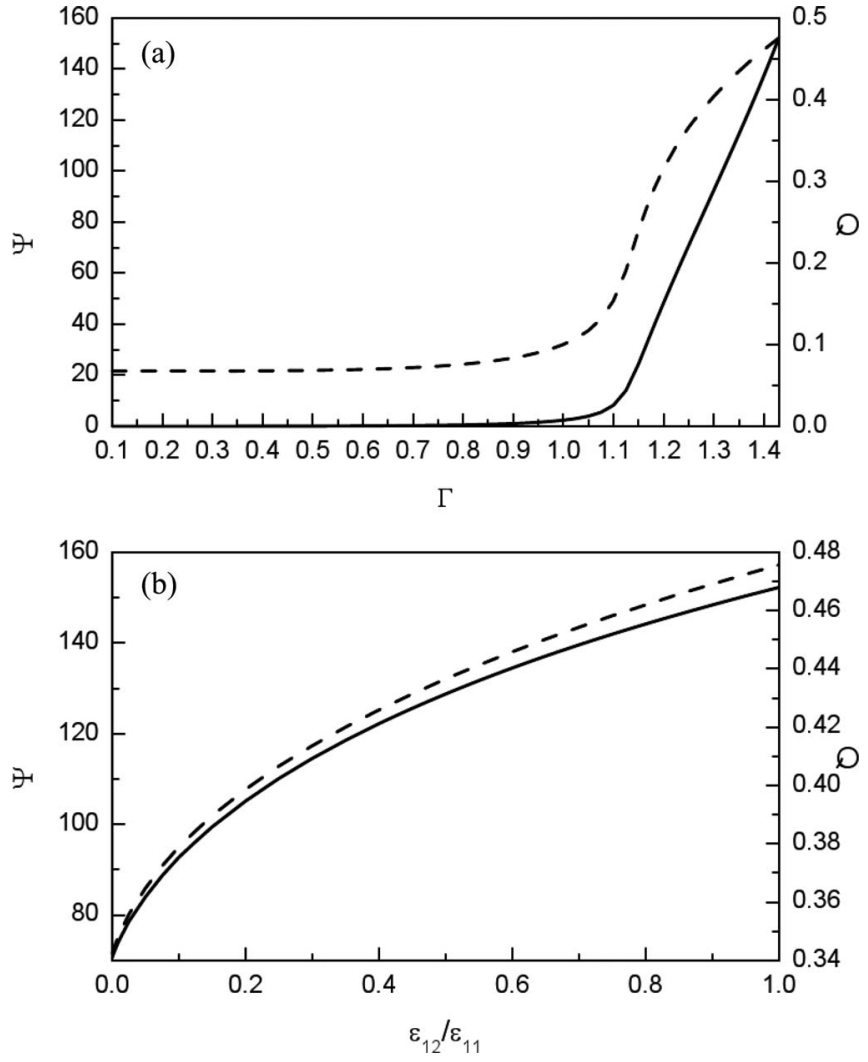

FIG. 10. Thermal path from the "molecular liquid" to the $G_{2}$ glass phase as calculated with the RY integral equation; $\Psi=h_{12}(x=0)$ (left-hand axis) and $Q$ (right-hand axis) are the order parameters. (a) Variation of $\Psi$ (full curve) and $Q$ (dashed line) with $\Gamma$ for a range parameter $c=0.5$ and an interreplica coupling $\varepsilon_{12}^{0}=\varepsilon$. (b) Variation of $\Psi$ (full curve) and $Q$ (dashed line) at $\Gamma=1.43$, upon decreasing $\varepsilon_{12}$ from 1 to 0 .

tions of the HNC or RY equations at the end of this two-step protocol are identical to those obtained by Protocols $\mathbf{a}$ and $\mathbf{b}$ for the end-points $\Gamma_{\mathrm{c} 1}$ and $\Gamma_{\mathrm{c} 2}$ of the two glass branches. The crucial advantage of Protocol $\mathbf{c}$ is that it provides a reversible thermodynamic path along which the excess free energy $f_{e x}$ can be calculated by thermodynamic integration, as detailed in Appendix B. In the case of the thermodynamically selfconsistent RY closure (11), such a thermodynamic integration procedure provides the only access to $f_{e x}$.

\section{EVIDENCE FOR A FIRST-ORDER TRANSITION TO AN IDEAL GLASS}

Using the theoretical and numerical tools presented in Secs. II-IV, we are now in a position to locate the hypothetical RFOT transition between the supercooled liquid $(L)$ and lowest free energy glass $\left(G_{2}\right)$ branches, by comparing the dimensionless free energies per particle

$$
f=f_{i d}+f_{e x}=\ln \left(\rho^{*}\right)-1+f_{e x}(\Gamma),
$$

of these two branches as functions of the reduced volume per particle $v^{*}=1 / \rho^{*}$ for fixed temperature $T^{*}$. The standard Maxwell double-tangent construction determines the volumes $v_{G_{2}}^{*}$ and $v_{L}^{*}$ of the two coexisting phases, since it ensures the equality of the pressures and chemical potentials of the two phases at coexistence. ${ }^{26}$ 


\section{A. HNC results}

Within the framework of HNC theory, the excess free energies are calculated using relation (17) for the excess chemical potential for both $L$ and $G_{2}$ phases, combined with the virial relation for the pressure; for the $L$ phase only the first term on the rhs contributes. The resulting free energies of the two phases are plotted versus $v^{*}$ in Fig. 11. The two curves are seen to cross at $v_{c r}^{*}=1.003$; above that value the free energy $f$ of the $L$ phase is the lower, while below that value, the free energy of the $G_{2}$ phase lies below that of the $L$ phase. The two free energy lines show little curvature and the slopes of the two curves at $v_{c r}^{*}$ are very close; consequently the double-tangent construction is a rather delicate task. The slopes $\left(\partial f / \partial v^{*}\right)_{T^{*}}$ of the two curves on both sides of their intersection are plotted in the inset of Fig. 11; they allow the volumes of the two phases to be estimated as $v_{G_{2}}^{*} \simeq 0.998$ and $v_{L}^{*} \simeq 1.007$, implying a very small relative volume change $\delta v^{*} / v_{c r}^{*}=\left(v_{L}^{*}-v_{G_{2}}^{*}\right) / v_{c r}^{*} \simeq 0.009$; for comparison, the relative volume change of freezing of the liquid into the FCC crystal is $\delta v^{*} / v_{f}^{*} \simeq 0.036 .{ }^{18,19}$ The RFOT may hence be considered as a weak first-order transition. The entropy change per particle at the transition follows from

$$
\begin{aligned}
\delta s= & \frac{S_{L}\left(v_{L}^{*}\right)-S_{G_{2}}\left(v_{G_{2}}^{*}\right)}{N k_{B}} \\
= & \ln \left(\frac{v_{L}^{*}}{v_{G_{2}}^{*}}\right)+\frac{\Gamma_{L}}{4} f_{e x, L}^{\prime}\left(\Gamma_{L}\right)-f_{e x, L}\left(\Gamma_{L}\right) \\
& -\frac{\Gamma_{G_{2}}}{4} f_{e x, G_{2}}^{\prime}\left(\Gamma_{G_{2}}\right)+f_{e x, G_{2}}\left(\Gamma_{G_{2}}\right) .
\end{aligned}
$$

Using the $\mathrm{HNC}$ results for the excess free energy $f_{\text {ex }}$ along the two branches, one obtains $\delta s=0.409$, which is again smaller than the corresponding value at freezing, which is typically of the order of 1.

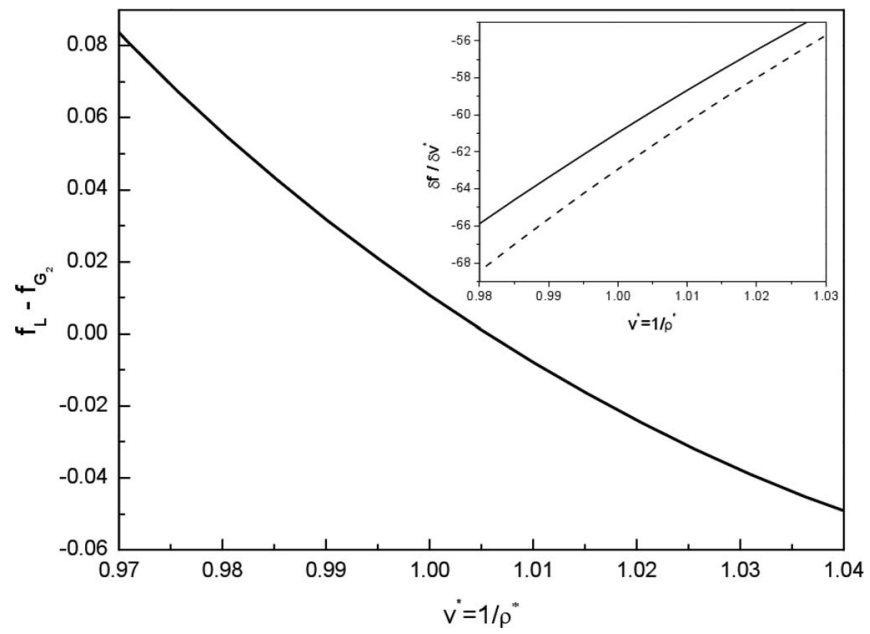

FIG. 11. HNC results for the difference of dimensionless free energy per particle $f=F /\left(N K_{B} T\right)$ in the liquid and in the $\mathrm{G}_{2}$ branch as a function of the reduced volume $v^{*}$. (Inset) Derivative of $f$ with respect to $v^{*}$ along the $L$ (dashed line) and $G_{2}$ (full line) branches. The data are plotted along the isotherm $T^{*}=0.1349$.

\section{B. RY results}

As emphasized earlier, Eq. (17) for $\mu_{e x}$ does not apply within the framework of the RY integral equation so that thermodynamic integration is required to calculate the excess free energies of the $L$ and $G_{2}$ phases. This is straightforward for the $L$ phase, for which Eq. (13) is used. In the case of the $G_{2}$ phase, the three-step Protocol $\mathbf{c}$ is used, as explained in Appendix $\mathrm{C}$ and illustrated in Fig. 10. The corresponding thermal path involves the "molecular liquid" phase characterized by a fixed value of the inter-replica coupling $\varepsilon_{12}$, comparable to the energy scale $\varepsilon$ in the soft-sphere potential (1).

The resulting total free energies (18) $f^{L}$ and $f^{G}$ of the two phases are plotted as functions of the dimensionless volume per particle $v^{*}$ in Fig. 12, for fixed $T^{*}=0.1349$ (corresponding to $\Gamma=1.65$ for $v^{*}=1$ ) down to $v^{*}=0.846$, the highest density for which a numerical solution of the RY equation was found along the $G_{2}$ branch; below that value, the iterative procedure did not converge. The free energy of the supercooled liquid phase lies consistently below the corresponding value of the glass phase $G_{2}$. Extrapolation of the data to lower values of $v^{*}$ suggests that the two free energy curves will intersect around $v_{c r}^{*}=0.82$, corresponding to $\Gamma_{c r}$ $=2.01$. Thus, the RFOT is predicted by RY theory to occur at a significantly larger value of the coupling compared to the HNC prediction $\Gamma_{c r}^{H N C}=1.65$. This difference is in keeping with the higher value of the Kauzmann coupling $\Gamma_{K}$ predicted by RY compared to HNC (cf. Eq. (16)). However, while $\Gamma_{c r}^{H N C}<\Gamma_{K}^{H N C}, \Gamma_{c r}^{R Y}>\Gamma_{K}^{R Y}$, which would imply that RFOT does not preempt the "Kauzmann catastrophe" (i.e., a negative complexity) within the framework of the RY integral equation.

This apparent failure of the RY theory is possibly due to an inaccurate description of the "molecular liquid" phase by the closure relation (11) for $g_{12}(r)$ when the choice $\alpha_{12}=+\infty$ is made. While the thermodynamic integration path is unaffected by this choice for the calculation of the free energy of the supercooled liquid phase $L$ (which involves a single

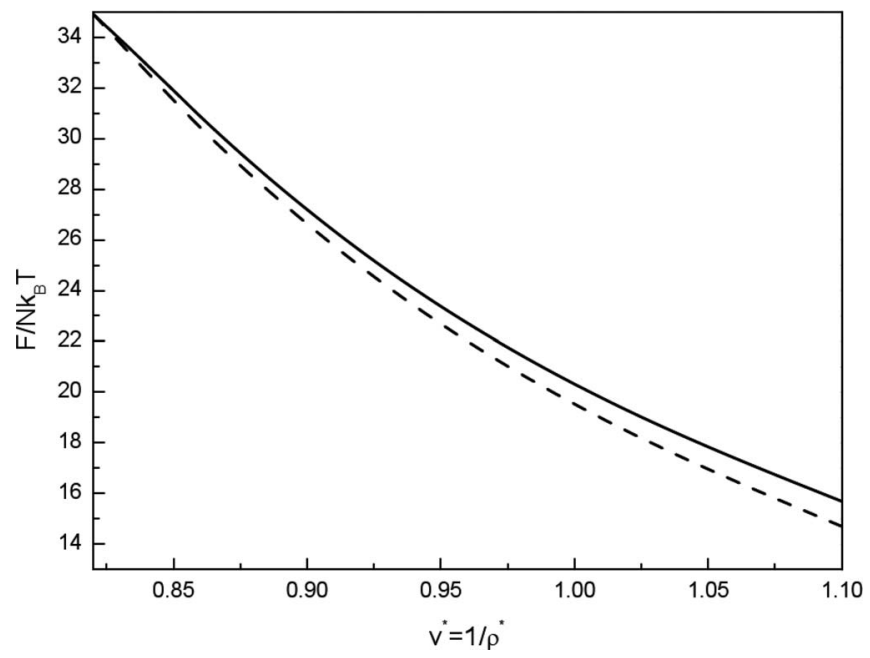

FIG. 12. RY results for $f=F /\left(N k_{B} T\right)$ at $T^{*}=0.1349$ as a function of the reduced volume $v^{*}$ along the $L$ (dashed line) and $G_{2}$ (full line) branches. The calculated curves are extrapolated from $v^{*}=0.846$ to smaller $v^{*}$, until they intersect. 
replica), the simplifying assumption $\alpha_{12}=+\infty$ no longer insures full thermodynamic self-consistency of the RY closure for the binary two-replicæ system, i.e., the "molecular liquid" phase which plays a central role within Protocol c. Thus, contrary to the HNC approach, where both $L$ and $G_{2}$ phases are treated on an equal footing through the use of relation (17) for the calculation of $\mu_{e x}$, the RY approach requires two different thermodynamic integration paths for the calculation of the free energies of the two phases. The lack of full thermodynamic self-consistency of the RY theory along the "molecular liquid" path may well result in an inaccurate estimate of the free energy difference between $L$ and $G_{2}$ branches, and hence of their intersection.

\section{CONCLUSIONS}

We have combined the HNC and RY closure relations (10) and (11) with the OZ relations (9) to determine the pair structure, thermodynamics and order parameters of a binary system of two coupled replicx of a soft-sphere fluid. Careful numerical solutions of the coupled integral equations for the pair distribution functions $g_{11}(r)$ and $g_{12}(r)$ over a wide range of coupling strengths $\rho^{*} / T^{* 1 / 4}$ predict the existence of three distinct branches of solutions.

The supercooled liquid $(L)$ branch is mapped out with the inter-replica coupling switched off $\left(\varepsilon_{12}=0\right)$. The configurational free energy and entropy (complexity) are calculated by subtracting an estimate of the vibrational contributions based on the classic expression for the Einstein frequency in dense fluids. The complexity is found to vanish at a Kauzmann temperature $T_{K}^{*}$ (or Kauzmann coupling $\Gamma_{K}$ ) the values of which are listed in Eq. (16) for HNC and RY.

Using three different search Protocols described in Sec. III, starting from an initial "molecular liquid," characterized by a non-zero value of the inter-replica coupling $\varepsilon_{12}$, two distinct glass branches are found in the limit $\varepsilon_{12} \rightarrow 0$. These two branches, labelled $G_{1}$ and $G_{2}$, are characterized by non-zero values of the order parameters $\Psi(\Gamma)=h_{12}(x=0 ; \Gamma)$ and $Q(\Gamma)-Q_{r}$ defined in Eq. (8); such "glass" solutions of the coupled HNC or RY integral equations cease to exist for $\Gamma<\Gamma_{c}$, where $\Gamma_{c}^{H N C}=1.58$ and $\Gamma_{c}^{R Y}=1.392$. The $G_{2}$ branch can be reasonably identified with the "ideal glass" phase predicted in a number of earlier papers, $3,10,12,14,15$ since the order parameters $\Psi(\Gamma)$ and $Q(\Gamma)$ increase with $\Gamma$. The opposite behaviour is observed along the $G_{1}$ branch, where $\Psi(\Gamma)$ and $Q(\Gamma)$ decrease with increasing $\Gamma$. A tentative explanation of this counterintuitive trend was put forward in Sec. IV.

The fact that the $G_{1}$ and $G_{2}$ branches are predicted by two different integral equations (HNC and RY) and the agreement of the integral equation approach for the $G_{2}$ branch with predictions from the replica symmetry theory not based on integral equations (small cage or harmonic resummation ${ }^{12}$ ) gives strong support to our belief that these branches are not a mere artifact of the integral equations, similar to the spurious solutions obtained with $\mathrm{HNC}$ near the liquid-gas transition. ${ }^{27}$ It would be interesting to check if the $G_{1}$ branch could also be found with methods not based on integral equations. The free energies of the thermodynamic states along the $G_{1}$ branch are systematically higher than those of the corresponding states on the $G_{2}$ branch which hence appears to correspond to the stable ideal glass.

Finally, the dimensionless free energies per particle, $f$ $=F /\left(N k_{B} T\right)$, were calculated along the branches $L$ and $G_{2}$ as functions of the reduced volume per particle $v^{*}$, for a fixed reduced temperature $T^{*}$. Within HNC theory, the excess free energies are directly accessible from the pair distribution functions $g_{11}(r)$ and $g_{12}(r)$ via Eq. (17) for the excess chemical potential $\mu_{e x}$, and the virial relation for the pressure. The resulting free energy curves shown in Fig. 11 intersect at $v_{c r}^{*}=1.003$, corresponding to $\Gamma_{c r}=1.65$. Note that due to the scaling properties of the soft-sphere systems with inverse power potential (1), $\Gamma_{c r}$ is independent of the choice of the isotherm $T^{*}$ along which a Maxwell doubletangent construction is carried out; the latter predicts a weak first-order RFOT between the $L$ and $G_{2}$ phases, characterized by small discontinuities $\delta v^{*}$ and $\delta s$ of the volume and entropy per particle. This finding appears to disagree with the earlier work of Mézard and Parisi, which predicts a RFOT with a continuous free energy, but a discontinuous order parameter $Q .^{10,12}$

Within the framework of the RY integral equation, the free energies of the $L$ and $G_{2}$ phases must be calculated by thermodynamic integration of the internal energy (Eq. (13)). This is a straightforward task for the $L$ branch which can be extended to arbitrarly low values of $\Gamma$. For the $G_{2}$ branch this is no longer true, since no solutions of the RY equations exist for $\Gamma<\Gamma_{c} \simeq 1.392$. Hence $G_{2}$ states at high $\Gamma$ must be connected to stable states of low $\Gamma$ via a "molecular liquid" phase characterized by a large inter-replica coupling $\varepsilon_{12} / \varepsilon \simeq 1$. This implies an asymmetry in the treatments of the $L$ and $G_{2}$ phases, which may explain that their free energy curves intersect only at an estimated value of $\Gamma_{c r}^{R Y} \simeq 2.01>$ $\Gamma_{K}^{R Y} \simeq 1.89$ (cf. Fig. 12). Possible reasons for this unphysical prediction are discussed at the end of Sec. V. However, the RY results confirm the weakly first order nature of the RFOT predicted by HNC theory. Future work will focus on a fully self-consistent version of the RY closure (11), involving a finite mixing parameter $\alpha_{12}$ as opposed to the simplified version used in the present work. More generally the adequacy of fluid integral equations for the description of the atypical "molecular phase" with a strong inter-replica coupling over a wide range of temperatures and densities requires a careful investigation.

Another open question concerns the nature of the counter-intuitive $G_{1}$ phase predicted by both HNC and RY integral equations, and its possible interference with the $L / G_{2}$ phase coexistence explored in the present work. A final objective of future work is the extension of our work to the case of Lennard-Jones systems, for which the RY integral equation must be replaced by the HMSA integral equation..$^{28}$

\section{ACKNOWLEDGMENTS}

The authors are grateful to Hugo Jacquin and Francesco Zamponi for helpful discussions. 


\section{APPENDIX A: CONFIGURATIONAL FREE ENERGY AND ENTROPY}

The dimensionless free energy per particle $f=F / N k_{B} T$ and entropy $s=S / N k_{B}$ can be split into ideal and excess parts, or into configurational and vibrational parts, as shown in Eq. (14). The configurational free energy $f_{\mathrm{c}}$ is hence given by

$$
\begin{gathered}
f_{\mathrm{c}}=f_{\mathrm{id}}+f_{\mathrm{ex}}-f_{\mathrm{v}}, \\
f_{\mathrm{id}}=\ln \left(\rho \Lambda^{3}\right)-1,
\end{gathered}
$$

where $\Lambda$ is the de Broglie thermal wavelength. The vibrational free energy results from the thermal oscillations of the atoms around long-lived equilibrium positions $\left\{\mathbf{X}_{i}\right\}$. In a quasi-harmonic approximation, each atom vibrates with a mean oscillation frequency $\omega_{0}$, and $f_{\mathrm{v}}$ is then trivially calculated from the partition function of a three-dimensional harmonic oscillator

$$
f_{\mathrm{v}}=F_{\mathrm{v}} / N k_{B} T=-\ln \left[\frac{1}{\Lambda^{3}}\left(\frac{2 \pi k_{B} T}{m \omega_{0}^{2}}\right)^{3 / 2}\right] .
$$

The square of the "Einstein" frequency $\omega_{0}$ for a disordered medium is determined by the mean square force acting on an atom (cf. Eq. (7.2.13) of Ref. 6),

$$
\omega_{0}^{2}=\frac{\rho}{3 m} \int \nabla^{2} v(r) g(r) d \mathbf{r} .
$$

Combining Eqs. (A1)-(A3), we arrive at the following expression for the configurational free energy:

$$
f_{\mathrm{c}}=f_{\mathrm{ex}}(\Gamma)+\ln \left[\rho\left(\frac{2 \pi k_{B} T}{m \omega_{0}^{2}}\right)^{3 / 2}\right]-1,
$$

from which the irrelevant de Broglie thermal wavelength is absent. Applying Eq. (A4) to the soft-sphere pair potential (1), and switching to the corresponding reduced variables, we find

$$
f_{\mathrm{c}}(\Gamma)=f_{\mathrm{ex}}(\Gamma)-\frac{3}{2} \ln \left[88 \Gamma^{4} I(\Gamma)\right]-1,
$$

where

$$
I(\Gamma)=\int_{0}^{\infty} g(x ; \Gamma) \frac{1}{x^{12}} d x
$$

and $x=r / \sigma$. In practice, remembering the scale invariance (4) of the pair distribution function, the integral in Eq. (A6) is conveniently calculated for $\rho^{*}=1$ and $\Gamma=\left(T^{*}\right)^{-1 / 4}$.

The configurational entropy (or complexity) $s_{\mathrm{c}}$ then follows from

$$
\begin{aligned}
s_{\mathrm{c}} & =-\left(\frac{\partial f_{\mathrm{c}}^{*}}{\partial T^{*}}\right)_{\rho^{*}}=-\left(\frac{\partial\left(T^{*} f_{\mathrm{c}}\right)}{\partial T^{*}}\right)_{\rho^{*}} \\
& =s_{\mathrm{ex}}-\frac{1}{2}+\frac{3}{2} \ln \left[88 \Gamma^{4} I(\Gamma)\right]-\frac{3}{8} \frac{\Gamma}{I(\Gamma)} \frac{d I(\Gamma)}{d \Gamma} .
\end{aligned}
$$

The variation of the reduced configurational free energy $f_{\mathrm{c}}^{*}$ with reduced temperature $T^{*}$ at fixed $\rho^{*}=1$ is shown in Fig. 2. $f_{\mathrm{c}}^{*}$ goes through a maximum at $T^{*}=T_{\mathrm{K}}^{*}$, where the configurational entropy $s_{\mathrm{c}}$ vanishes ("Kauzmann catastrophe").

\section{APPENDIX B: HNC RELATION FOR THE EXCESS CHEMICAL POTENTIAL FOR THE TWO-REPLICAE SYSTEM}

We consider the symmetric two-replicæ system defined in Sec. II. The excess chemical potential is the change in grand potential $\Omega$ upon adding an atom to one of the replicæ, say replica 1. Invoking translational invariance the additional atom may be assumed to be placed at the origin; it acts like an external potential $\varphi_{1}(r)=v_{11}(r)=v(r)$ on atoms of replica 1 , and $\varphi_{2}(r)=v_{12}(r)=-\varepsilon_{12} w(r)$ on atoms of replica 2 . The binary system is now inhomogeneous and characterized by the two one-particle densities $\rho_{1}(\mathbf{r})$ and $\rho_{2}(\mathbf{r})$ associated with the two replicæ, and the grand potential becomes a functional $\Omega_{\varphi_{1}, \varphi_{2}}\left[\rho_{1}(\mathbf{r}), \rho_{2}(\mathbf{r})\right]$ of these local densities. $\Omega_{\varphi_{1}, \varphi_{2}}$ may be expanded in a functional Taylor expansion in the deviations $\Delta \rho_{\alpha}(\mathbf{r})=\rho_{\alpha}(\mathbf{r})-\rho_{0}$ of these local densities from their bulk value $\rho_{0}$ in the absence of the external potentials due to the added particle. Truncating the expansion after second order (HNC approximation) one arrives at the following expression (cf. Sec. 4.3 of Ref. 6):

$$
\begin{aligned}
\Delta \Omega= & \Omega_{\varphi_{1}, \varphi_{2}}\left[\rho_{1}(\mathbf{r}), \rho_{2}(\mathbf{r})\right]-\Omega_{0} \\
= & \sum_{\alpha=1}^{2}\left\{\int \rho_{\alpha}(\mathbf{r}) \varphi_{\alpha}(\mathbf{r}) d \mathbf{r}\right. \\
& \left.+k_{B} T \int\left[\rho_{\alpha}(\mathbf{r}) \ln \frac{\rho_{\alpha}(\mathbf{r})}{\rho_{0}}-\Delta \rho_{\alpha}(\mathbf{r})\right] d \mathbf{r}\right\} \\
& -\frac{k_{B} T}{2} \sum_{\alpha=1}^{2} \sum_{\beta=1}^{2} \iint \Delta \rho_{\alpha}(\mathbf{r}) c_{\alpha \beta}^{(0)}\left(\left|\mathbf{r}-\mathbf{r}^{\prime}\right|\right) \Delta \rho_{\beta}\left(\mathbf{r}^{\prime}\right) d \mathbf{r} d \mathbf{r}^{\prime},
\end{aligned}
$$

where $\Omega_{0}$ is the grand potential of the homogeneous binary system (i.e., in the absence of the additional particle at the origin), and the $c_{\alpha \beta}^{(0)}(r)$ are the corresponding direct correlation functions. $\Delta \Omega$ is then minimized with respect to the local densities, leading to the two coupled Euler-Lagrange equations yielding the equilibrium one-particle densities:

$$
\begin{aligned}
\rho_{1}(\mathbf{r})= & \rho_{0} \exp \left\{-\beta \varphi_{1}(\mathbf{r})+\int c_{11}\left(\left|\mathbf{r}-\mathbf{r}^{\prime}\right|\right) \Delta \rho_{1}\left(\mathbf{r}^{\prime}\right) d \mathbf{r}^{\prime}\right. \\
& \left.+\int c_{12}\left(\left|\mathbf{r}-\mathbf{r}^{\prime}\right|\right) \Delta \rho_{2}\left(\mathbf{r}^{\prime}\right) d \mathbf{r}^{\prime}\right\}, \\
\rho_{2}(\mathbf{r})= & \rho_{0} \exp \left\{-\beta \varphi_{2}(\mathbf{r})+\int c_{12}\left(\left|\mathbf{r}-\mathbf{r}^{\prime}\right|\right) \Delta \rho_{1}\left(\mathbf{r}^{\prime}\right) d \mathbf{r}^{\prime}\right. \\
& \left.+\int c_{22}\left(\left|\mathbf{r}-\mathbf{r}^{\prime}\right|\right) \Delta \rho_{2}\left(\mathbf{r}^{\prime}\right) d \mathbf{r}^{\prime}\right\} .
\end{aligned}
$$

These one-particle densities are then substituted into the expression (B1) for $\Delta \Omega$, and use is made of Percus' identification $^{6}$

$$
\begin{aligned}
& \rho_{1}\left(\mathbf{r} \mid \varphi_{1}\right)=\rho_{0} g_{11}^{(0)}(r), \\
& \rho_{2}\left(\mathbf{r} \mid \varphi_{2}\right)=\rho_{0} g_{12}^{(0)}(r),
\end{aligned}
$$


where $g_{\alpha \beta}^{(0)}(r)$ are the pair distribution functions of the symmetric, homogeneous reference mixture (where $g_{22}^{(0)}(r)$ $\left.\equiv g_{11}^{(0)}(r)\right)$. We henceforth drop the superscript (0) for the correlation functions of the homogeneous reference system, and make use of the two coupled OZ relations:

$$
\begin{aligned}
& \gamma_{11}(r) \equiv h_{11}(r)-c_{11}(r)=\rho\left[c_{11} \otimes h_{11}+c_{12} \otimes h_{12}\right], \\
& \gamma_{12}(r) \equiv h_{12}(r)-c_{12}(r)=\rho\left[c_{11} \otimes h_{12}+c_{12} \otimes h_{11}\right],
\end{aligned}
$$

where $\otimes$ denotes a convolution product. Gathering Eqs. (B1)(B6) (with $\rho=\rho_{0}$ ), we arrive at the HNC relation for the chemical potential (Eq. (17)) $\beta \mu_{e x}=\beta \Delta \Omega$ (written in reduced units). This result is a special case of the general HNC relation for the excess chemical potentials $\mu_{e x}^{\alpha}(1 \leq \alpha \leq n)$ of the $n$ species of an $n$-component mixture. ${ }^{25}$

The important point for the case of two weakly coupled replicæ is that the expression (17) does not depend explicitly on the coupling $\varepsilon_{12}$ between the two replicæ, but only implicitly through the correlation functions $h_{11}(r), c_{11}(r)$ and $h_{12}(r), c_{12}(r)$, which are calculated within the HNC approximation of Sec. III. In particular, Eq. (17) remains valid in the limit $\varepsilon_{12} \rightarrow 0$. In the supercooled liquid phase, $h_{12}(r)$ $=c_{12}(r)=0$ in that limit, so that relation (17) reduces to its one-component version. However in the glass phases $G_{1}$ and $G_{2}, h_{12}(r)$ and $c_{12}(r)$ as calculated by solving the coupled $\mathrm{HNC}$ integral equations, are non-zero in the limit $\varepsilon_{12} \rightarrow 0$, so that the full expression (17) applies in that case.

\section{APPENDIX C: EXCESS FREE ENERGY OF THE IDEAL GLASS BY THREE-STEP THERMODYNAMIC INTEGRATION}

As explained in Sec. IV, the excess chemical potential $\mu_{e x}$ and free energy $f_{e x}$ of the glass phases $G_{1}$ and $G_{2}$ is directly accessible within HNC theory via relation (17). This is no longer true within the framework of the RY integral equation, and one must resort to a reversible thermodynamic path within Protocol c which links the glass phases to the supercooled "molecular liquid" phase. Such a path involves three successive steps. For convenience we set the reduced number density $\rho^{*}=1$, so that $\Gamma=\left(T^{*}\right)^{-1 / 4}$. We consider a binary "two replicæ" system throughout, where atoms of the opposite replicæ interact via the attractive potential (5).

(1) Step 1 is carried out in the "molecular liquid" phase, for a fixed value of the inter-replica coupling $\varepsilon_{12}$. A straightforward generalization of Eq. (13) relates the excess free energy at $\Gamma=\Gamma_{1}$ to that of a reference state $\Gamma_{0}$ via

$$
f_{e x}\left(\Gamma_{1}, \varepsilon_{12}\right)=f_{e x}\left(\Gamma_{0}, \varepsilon_{12}\right)+4 \int_{\Gamma_{0}}^{\Gamma_{1}} u_{e x}\left(\Gamma, \varepsilon_{12}\right) \frac{d \Gamma}{\Gamma},
$$

where $u_{e x}$ is the dimensionless excess internal energy, and $\Gamma_{0}$ is chosen to be sufficiently small for HNC to be quantitatively accurate, so that $f_{\mathrm{ex}}\left(\Gamma_{0}, \varepsilon_{12}\right)$ may be calculated via the relation (17) for $\mu_{e x}$ and the standard virial relation for $\Pi_{e x}(\Gamma)\left(f_{e x}\right.$ $\left.=\Pi_{e x}-\beta \mu_{e x}\right)$.
(2) In step 2, for a fixed value $\Gamma_{1}$, the inter-replica coupling is progressively reduced from $\varepsilon_{12}$ to 0 . The corresponding variation in excess free energy is given by

$$
\begin{aligned}
\left(\frac{\partial f_{\mathrm{ex}}}{\partial \lambda}\right)_{\Gamma_{1}} & =-4 \pi \rho_{1}^{*} \beta_{1}^{*} \int_{0}^{\infty} g_{12}\left(x ; \Gamma_{1} ; \lambda\right) w(x) x^{2} d x \\
& =-\beta_{1}^{*} Q\left(\Gamma_{1}, \lambda\right) \quad\left(0 \leq \lambda \leq \varepsilon_{12}\right),
\end{aligned}
$$

where $Q$ is the overlap order parameter (8) and $\rho_{1}^{*} \beta_{1}^{*}=\Gamma_{1}^{4}$. A standard Debye-Kirkwood coupling constant integration of both sides of Eq. (C3) leads to

$$
f_{e x}\left(\Gamma_{1}, \varepsilon_{12}=0\right)=f_{e x}\left(\Gamma_{1}, \varepsilon_{12}\right)+\Gamma_{1}^{4} \int_{0}^{\varepsilon_{12}} Q\left(\Gamma_{1}, \lambda\right) d \lambda .
$$

Depending on the choice of $\Gamma_{1}$, this two-step path leads us to the glass branches $G_{1}$ or $G_{2}$ (cf. Sec. IV and Fig. 10).

(3) Step 3 maps out the branches $G_{1}$ or $G_{2}$ by keeping $\varepsilon_{12}=0$ and varying $\Gamma$. The excess free energy is finally given by another application of thermodynamic integration (Eq. (13)),

$$
\begin{aligned}
f_{e x}\left(\Gamma_{2}, \varepsilon_{12}=0\right)= & f_{e x}\left(\Gamma_{1}, \varepsilon_{12}=0\right) \\
& +4 \int_{\Gamma_{1}}^{\Gamma_{2}} u_{e x}\left(\Gamma, \varepsilon_{12}=0\right) \frac{d \Gamma}{\Gamma} .
\end{aligned}
$$

Protocol c, with a sufficiently large range parameter $c(\gtrsim 0.5)$ provides a continuous thermodynamic path leading to the excess free energy $f_{e x}(\Gamma)$ of the glass branches $G_{1}$ and $G_{2}$.

${ }^{1}$ See, e.g., C. A. Angell, J. Non-Cryst. Solids 131-133, 13 (1991).

${ }^{2}$ P. G. Debenedetti and F. H. Stillinger, Nature (London) 410, 259 (2001).

${ }^{3}$ For a collection of reviews of recent works, see Structural Glasses and Supercooled Liquids: Theory, Experiment and Applications, edited by P. G. Wolynes and V. Lubchenko (John Wiley, Hoboken, 2012); see also L. Berthier and G. Biroli, Rev. Mod. Phys. 83, 587 (2011).

${ }^{4}$ W. Götze, Complex Dynamics of Glass-forming Liquids: A Mode-Coupling Theory (Oxford University Press, Oxford, 2009).

${ }^{5}$ C. A. Angell, J. Phys. Chem. Solids 49, 863 (1988).

${ }^{6}$ J.-P. Hansen and I. R. McDonald, Theory of Simple Liquids, 4th ed. (Elsevier, Amsterdam, 2013).

${ }^{7}$ A. W. Kauzmann, Chem. Rev. 43, 219 (1948).

${ }^{8}$ G. Adam and J. H. Gibbs, J. Chem. Phys. 43, 139 (1965); for a recent reassessment of Adam-Gibbs theory, see J. C. Dyre, T. Hechsher, and K. Niss, J. Non-Cryst. Solids 355, 624 (2009).

${ }^{9}$ For a recent discussion of possible topologies of the free energy landscapes of supercooled liquids and glasses, see P. Charbonneau, J. Kurchan, G. Parisi, P. Urbani, and F. Zamponi, Nat. Commun. 5, 3725 (2014).

${ }^{10}$ M. Mézard and G. Parisi, J. Phys. A: Math. Gen. 29, 6515-6524 (1996).

${ }^{11}$ S. Franz and G. Parisi, Physica A 261, 317 (1998).

${ }^{12}$ M. Mézard and G. Parisi, J. Chem. Phys. 111, 1076 (1999).

${ }^{13}$ T. Morita and K. Hiroike, Prog. Theor. Phys. 25, 537 (1961).

${ }^{14}$ B. Coluzzi, M. Mézard, G. Parisi, and P. Verrochio, J. Chem. Phys. 111, 9039 (1999).

${ }^{15}$ G. Parisi and F. Zamponi, Rev. Mod. Phys. 82, 789 (2010).

${ }^{16}$ F. J. Rogers and D. A. Young, Phys. Rev. A 30, 999 (1984).

${ }^{17}$ J.-M. Bomont, G. Pastore, and J.-P. Hansen, EuroPhys. Lett. 105, 36003 (2014).

${ }^{18}$ See, e.g., J.-P. Hansen, Phys. Rev. A 2, 221 (1970).

${ }^{19}$ W. G. Hoover et al., J. Chem. Phys. 52, 4931 (1970).

${ }^{20}$ J. N. Cape and L. V. Woodcock, J. Chem. Phys. 72, 976 (1980).

${ }^{21}$ J. Habasaki and A. Ueda, J. Chem. Phys. 138, 144503 (2013).

${ }^{22}$ J.-M. Bomont, Adv. Chem. Phys. 139, 1 (2008). 
${ }^{23}$ M. J. Gillan, Mol. Phys. 38, 1781 (1979).

${ }^{24}$ G. M. Abernethy and M. J. Gillan, Mol. Phys. 39, 839 (1980).

${ }^{25}$ L. Verlet and D. Levesque, Physica 28, 1124 (1962); J.-P. Hansen, G. M. Torrie, and P. Vieillefosse, Phys. Rev. A 16, 2153 (1977).
${ }^{26}$ See, e.g., D. Chandler, Introduction to Modern Statistical Mechanics (Oxford University Press, New York, 1987).

${ }^{27}$ L. Belloni, J. Chem. Phys. 98, 8080 (1993).

${ }^{28}$ G. Zerah and J.-P. Hansen, J. Chem. Phys. 84, 2336 (1986). 